\title{
Data Preparation 101: How To Use Query- By-Example To Get Your Research Dataset Ready For Primetime
}

Paul J. Lazarony, California State University, Northridge, USA

Donna A. Driscoll, California State University, Northridge, USA

\begin{abstract}
Researchers are often distressed to discover that the data they wanted to use in their landmark study is not configured in a way that is usable by a Statistical Analysis Software Package (SASP). For example, the data needed may come from two or more sources and it may not be clear to the researcher how to get them combined into one analyzable dataset. Fortunately, there is hope. The query facility within database management software (DBMS), such as Microsoft Access, is particularly well-equipped to do the data reconfigurations necessary to get datasets ready for a SASP. Unfortunately, most researchers have either never been exposed to a DBMS or are unaware of the powerful data-transforming queries that they can perform. The purpose of this paper is to introduce researchers to some very helpful and relatively easy to learn techniques for solving common dataset misconfiguration problems. It is presented in three sets of hands-on examples: (1) how to import a dataset from an electronic spreadsheet into a database table, (2) how to use an easy-to-learn DBMS facility called Query-By-Example (QBE) to perform specific data reconfiguration tasks, and (3) how to import the reconfigured dataset from the DBMS into a SASP. The software used is MS Excel, MS Access, and SPSS (originally called Statistical Package for the Social Sciences). The examples are presented step-by-step so that the reader can follow along using files downloadable from the authors' website
\end{abstract}

Keywords: Database; Dataset; Query; Research; Spreadsheet; Statistical Analysis

\section{INTRODUCTION}

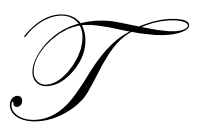

he authors of this paper are information systems faculty who have discovered that database techniques can be very helpful in dealing with common problems associated with research datasets. This paper is the second in a series that attempts to make these techniques more accessible to researchers in other fields.

Reconfiguration of datasets in a database can be approached in two basic ways: (a) Structured Query Language (SQL) and (b) Query-By-Example (QBE). SQL is a high-level computer language that involves writing computer code to do queries of a database; QBE allows the user to formulate queries using a graphical user interface (which allows the use of dropdown menus, toolbars, and wizards) and is thus much more user-friendly.

Our first paper in this series focused on the first of these approaches. Specifically, we used SQL to efficiently correct, recode, and reorganize a dataset downloaded from one table that was embedded in a web page. The current paper focuses on the second approach. Specifically, we will demonstrate how to use QBE to combine data stored in multiple tables in a variety of useful ways.

This paper is presented in three phases, each of which includes hands-on examples. In Phase I, we demonstrate the steps required to import a dataset stored in an MS Excel file into an MS Access database table. Then, in Phase II, we take the reader through a series of five examples that illustrate how to combine data that exist 
in multiple tables. Finally, in Phase III, we show how to import the newly combined dataset from an MS Access table into SPSS for statistical analysis.

As previously mentioned, this example is designed so that the reader may follow along step-by-step. The Web page containing the data can be found at www.csun.edu/ pj126399/dataprep101. The following software will also be required to complete the example: MS Excel, MS Access, and SPSS. If SPSS is not available, Phase III could also be accomplished using another statistical analysis package such as SAS or Minitab. The dataset could also be returned to MS Excel for statistical analysis.

\section{PHASE I: IMPORTING A DATASET FROM MS EXCEL INTO AN MS ACCESS TABLE}

Although datasets could come to a researcher in a variety of forms, one of the most commonly used current formats is an MS Excel file. Depending on the particular dataset and the complexity of the required analysis, a researcher may sometimes choose to leave the data in MS Excel and simply do the analysis right there. At other times, however, it could be difficult to use the data in its original form. The following steps illustrate the ease of importing an MS Excel file into an MS Access table:

A. Download the DataPrep101.mdb and 00_SalesData.xls files from the Web page shown above. Figure 1 shows the files after download. Be sure to save them in a new folder on your computer's hard drive, named DataPrep101.

B. Open the 00_SalesData.xls in MS Excel. The file should look like Figure 2 below:

Figure 1: Files to Download for this Example

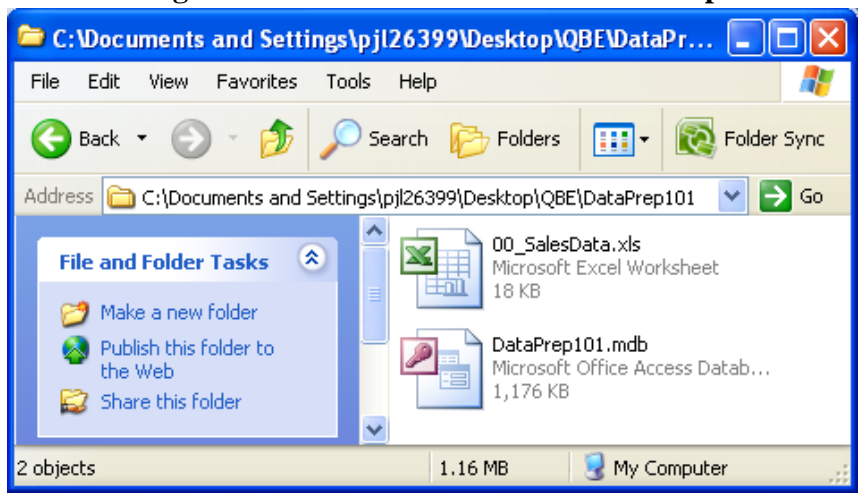

Figure 2: 00_SalesData.xls File Opened in MS Excel

\begin{tabular}{|c|c|c|c|c|c|c|c|}
\hline \multicolumn{7}{|c|}{ [ Microsoft Excel - 00_SalesData.xls } & \multirow{2}{*}{$\begin{array}{r}\square \square x \\
-5 x\end{array}$} \\
\hline \multicolumn{7}{|c|}{ :凶] File Edit Wiew Insert Format Iools } & \\
\hline \multicolumn{8}{|c|}{ 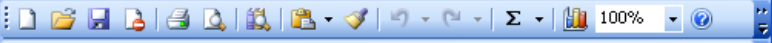 } \\
\hline \multirow{2}{*}{\multicolumn{2}{|c|}{$\begin{array}{l}\text { Calibri } \\
\text { B12 }\end{array}$}} & \multirow{2}{*}{\multicolumn{6}{|c|}{ 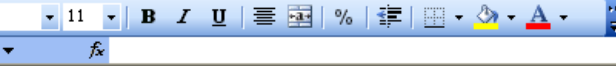 }} \\
\hline & & & & & & & \\
\hline \multicolumn{2}{|r|}{ A } & B & C & D & E & $\mathrm{F}$ & G \\
\hline 1 & SalesID & Date & State & Region & SalesRep & Gender & Amount \\
\hline 2 & 12101 & $7 / 3 / 2009$ & CA & w & 66 & M & $\$ 5,000.00$ \\
\hline 3 & 12102 & 7/4/2009 & $\mathrm{OH}$ & $\mathrm{E}$ & 33 & $\mathrm{~F}$ & $\$ 10,000.00$ \\
\hline 4 & 12103 & $7 / 5 / 2009$ & GA & $\mathrm{E}$ & 22 & M & $\$ 5,500.00$ \\
\hline 5 & 12104 & $7 / 6 / 2009$ & WI & w & 44 & M & $\$ 3,300.00$ \\
\hline 6 & 12105 & 7/7/2009 & CA & w & 55 & $\mathrm{~F}$ & $\$ 4,400.00$ \\
\hline & $M \backslash S h$ & eet1/Shee & 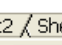 & & & & $>1$ \\
\hline Rea & & & & & & NUM & \\
\hline
\end{tabular}

C. The goal is to import the 00_SalesData.xls spreadsheet file into a table in the DataPrep101.mdb database file. To complete this task, follow the steps below:

1. Open the DataPrep101.mdb file in MS Access. Your screen will look similar to Figure 3 below. Notice the Tables and Queries listed in on the left-hand side of the screen. We will be using these objects later in this paper (in Phase II).

2. Notice the Security Warning in the middle of the screen. Click the Options button. The Microsoft Office Security Options dialog box will appear. See Figure 4.

3. The default is to leave the database file as read-only. Since changes to the database will be necessary, will need to enable all content. Click the radio button next to the Enable this content option. Then click $\mathbf{O K}$. You will need to repeat these steps ( $2 \& 3$ ) each time you open the database file.

4. To import the 00_SalesData.xls spreadsheet file, click the External Data tab, then click the Excel button on the toolbar. The Get External Data-Excel Spreadsheet dialog box will appear. See Figure 5. 
Figure 3: DataPrep101.mdb File Opened in MS Access

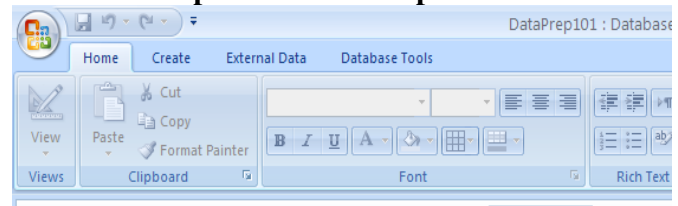

Security Warning Certain content in the database has been disabled Options...

\section{All Access Objects}

Tables

T1_CensusData

团 01_StoreSalesByzipcode

02_Gradesfall2008

圈 02_Gradesspring2008

03-GradePts

圈 04_StudentsFall2008

国 04_StudentsSpring2009

团 05_Employees

畨 06_MissedClassesAndPointsEarned

Queries

06_MissedClassesAndPointsEarned Queng
Figure 4: Microsoft Office Security Dialog Box

Microsoft Office Security Options
Security Alert
VBA Macro
Access has disabled potentially harmful content in this database.
If you trust the contents of this database and would like to enable it for this session
only, click Enable this content.
Warning: It is not possible to determine that this content came from a
trustworthy source. You should leave this content disabled unless the
content provides critical functionality and you trust its source.
More information
File Path: c: Documents and Settings bji2 26399 Pesktop lQBE DataPrep 101.mdb
O Help protect me from unknown content (recommended)
(E) Enable this content:

Figure 5: Database Window with Get External Data-Excel Spreadsheet Dialog Box

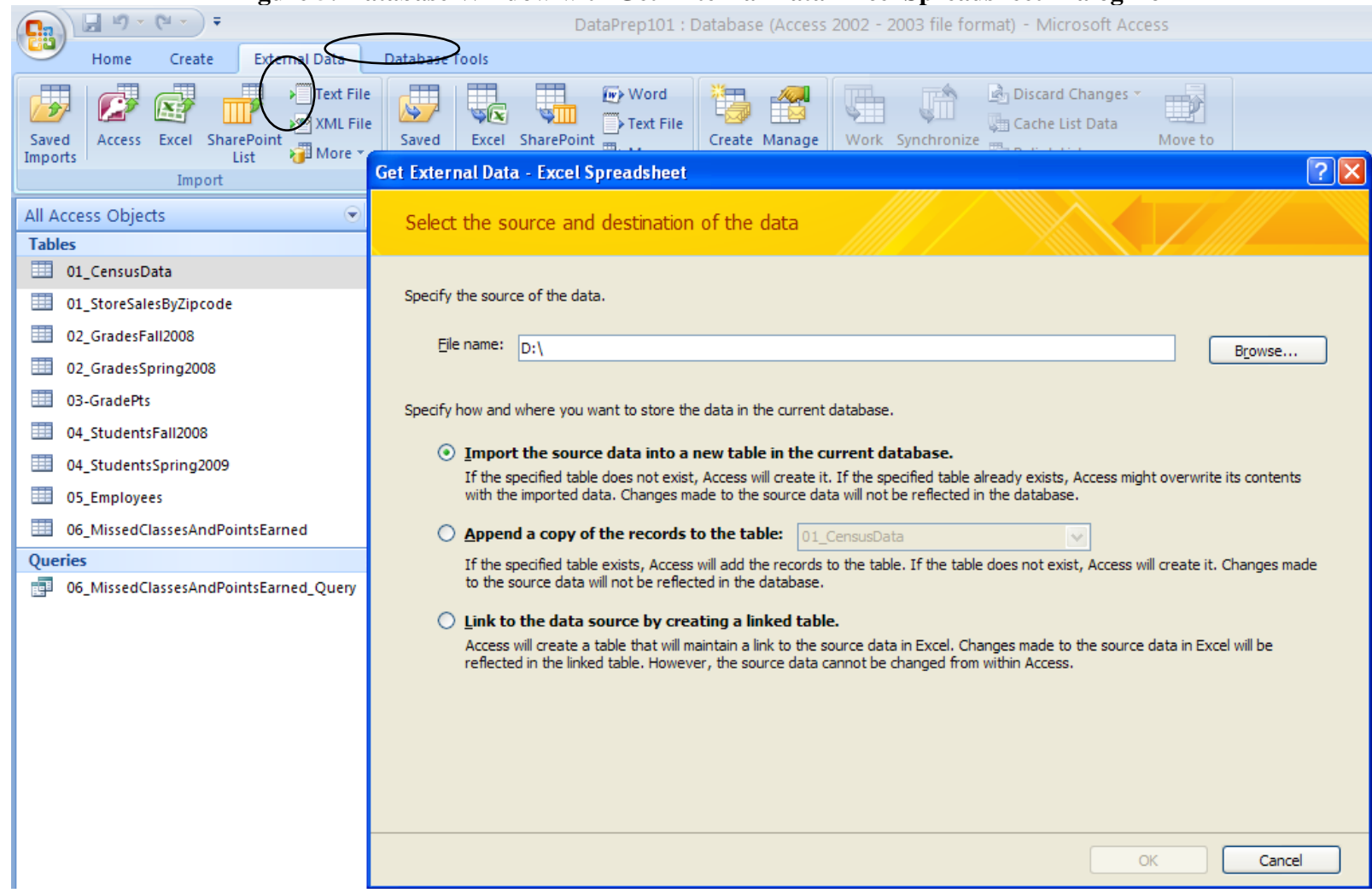

5. Click the Browse button. Locate the 00_SalesData.xls spreadsheet file; click Open. The Get External Database dialog box should now look similar to Figure 6.

6. Leave the radio button checked next to Import the source data into a new table in the current database. Click OK. The Import Spreadsheet Wizard will appear (see Figure 7). 
Figure 6: Get External Data Dialog Box-Screen 2

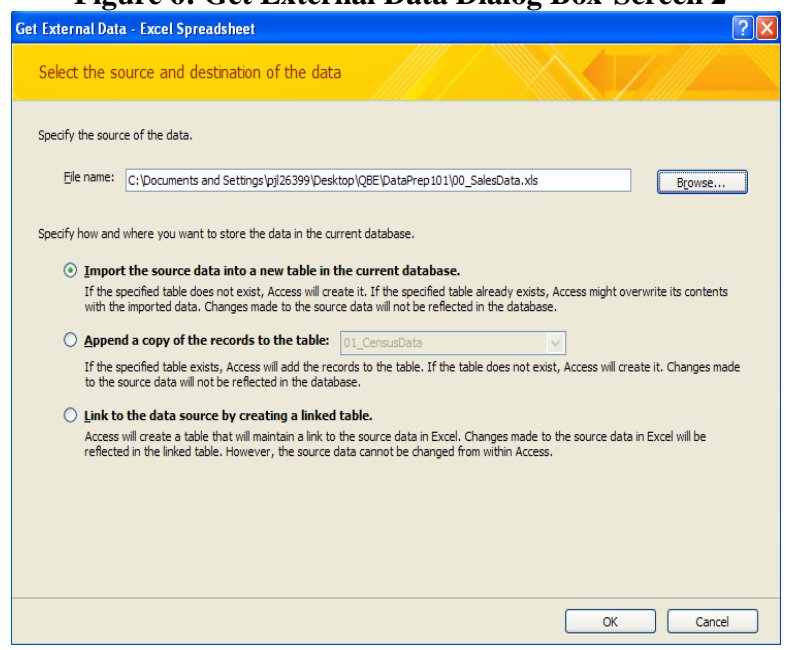

Figure 7: Import Spreadsheet Wizard-Screen 1

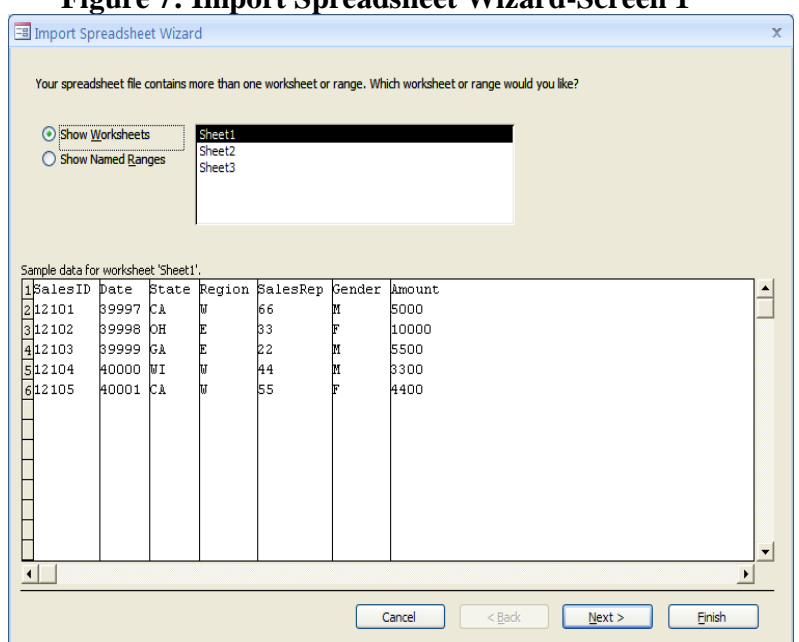

7. Notice that the all of the column headings and data from the 00_SalesData.xls spreadsheet file are displayed in the wizard. Click Next. The second screen of the wizard appears (see Figure 8).

8. This second screen assumes that the first row of data from our spreadsheet file contains column headings. Since this assumption is correct, leave the checkmark next to First Row Contains Column Headings. Click Next. The third screen of the wizard appears (see Figure 9).

Figure 8: Import Spreadsheet Wizard-Screen 2

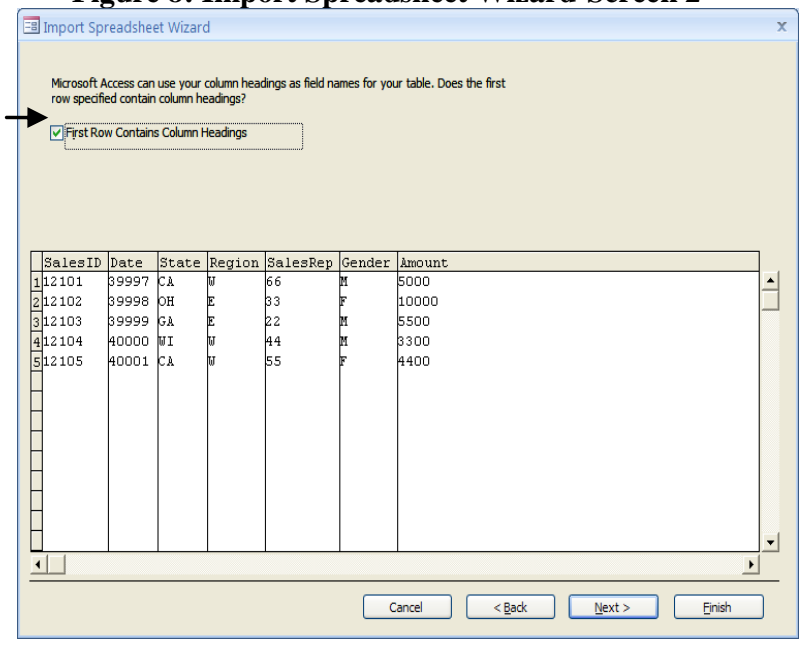

Figure 9: Import Spreadsheet Wizard-Screen 3

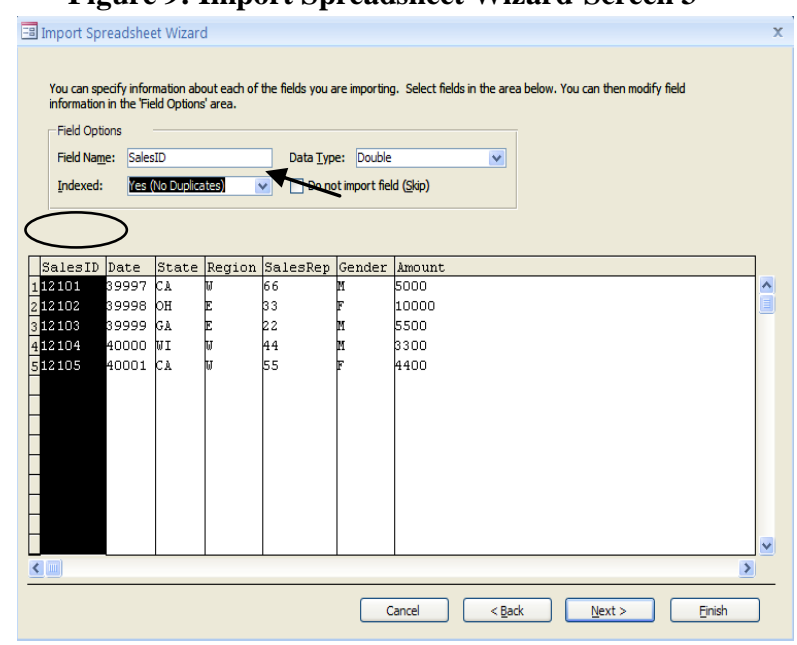

9. This third screen of the wizard allows you to change the data type of each field (column), if needed, as well as indicate how the table will be indexed.. In our example, the only change we need is to have the SalesID column be set as a unique key field (so that it might later be used to link to a SalesID field in another table). To accomplish this, first click on the SalesID column heading. Second, click the down arrow next to Indexed and select Yes (No Duplicates). Click Next. The fourth screen of the wizard appears (see Figure 10).

10. Click the radio button next to Choose my own primary key. This will select the SalesID as the primary key (or unique field) for this table. Click Next. The fifth and final screen of the wizard appears (see Figure 11). 
Figure 10: Import Spreadsheet Wizard-Screen 4

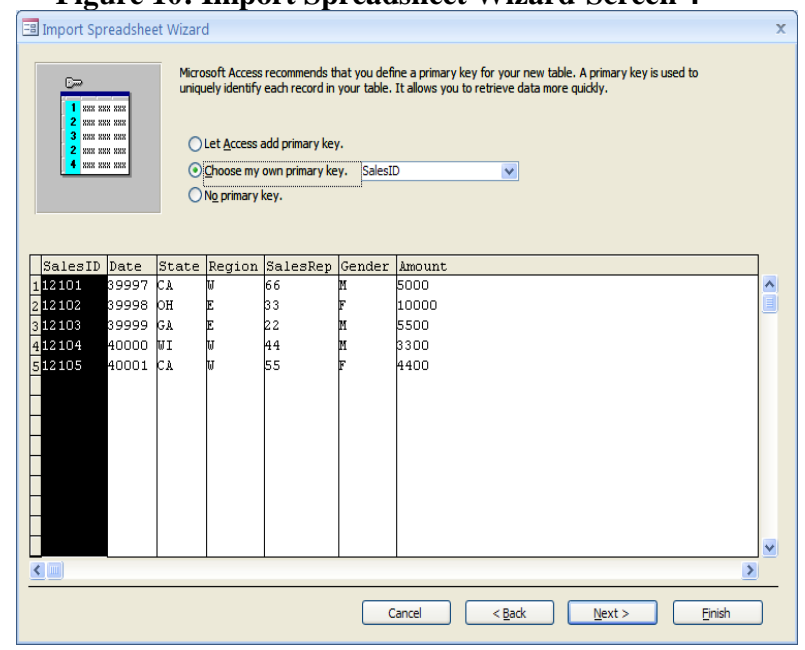

Figure 11: Import Spreadsheet Wizard-Screen 5

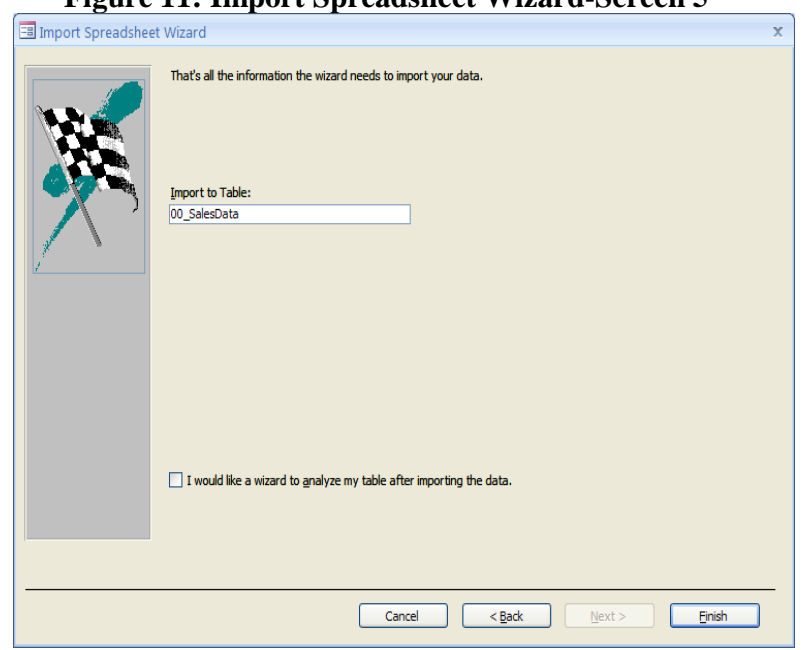

11. In the Import to Table text box, type in the new table name: 00_SalesData. Click Finish. In the next dialog box, click Close. Congratulations, the 00_SalesData table now appears in the list of tables of the left-side of the screen. Double click the new table name (00_SalesData) and the new table appears on the right (see Figure 12).

Figure 12: Database File Showing the Newly Imported 00_SalesData Table and Dataset

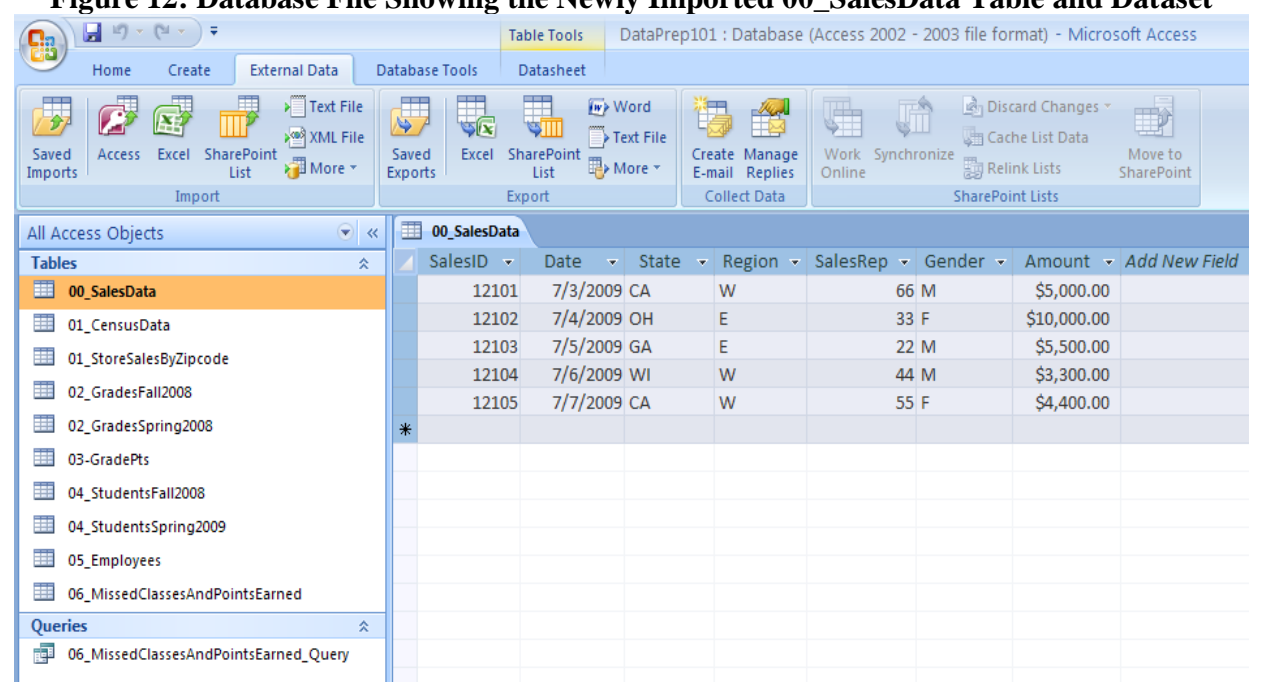

12. To close the table, just press the $\mathbf{X}$ in the upper right corner of the table.

13. Phase I is now complete. The reader may now continue on to Phase II or close the DataPrep101.mdb file.

\section{PHASE II: FIVE EXAMPLES OF HOW TO USE MS ACCESS' QUERY BY EXAMPLE TO RECONFIGURE DATASETS}

Although the QBE facility in MS Access can be used to handle most types of data misconfigurations, some situations tend to occur fairly frequently and, unfortunately, without the use of such database techniques, can be either complete barriers to further progress on a project or a problem that ends up being dealt with by the brute force approach of fixing the problems by hand at a substantial cost in time, money, or both. 
In Phase II, we focus on five relatively common data problems that can be solved using QBE in a tiny fraction of the time it would take to do it the old-fashioned (and labor-intensive) way:

A. combining the columns from two related tables (using a JOIN query),

B. adding the rows in one table into another table (using an APPEND query),

C. recoding a column in one table (using data stored in a second CONVERSION table),

D. dealing with tables having overlapping data (using UNMATCHED and APPEND queries), and

E. allowing the matching of values in one column that reference the values of another column in the same table (using a SELF-JOIN query).

These five situations are summarized in Table 1, along with a simple example of each that will then be described in detail. The reader is encouraged to work through these examples using the same database available from our website. (Please see the Introduction section of this paper for the file location.)

Table 1: Five Database Problems that can be Solved using QBE

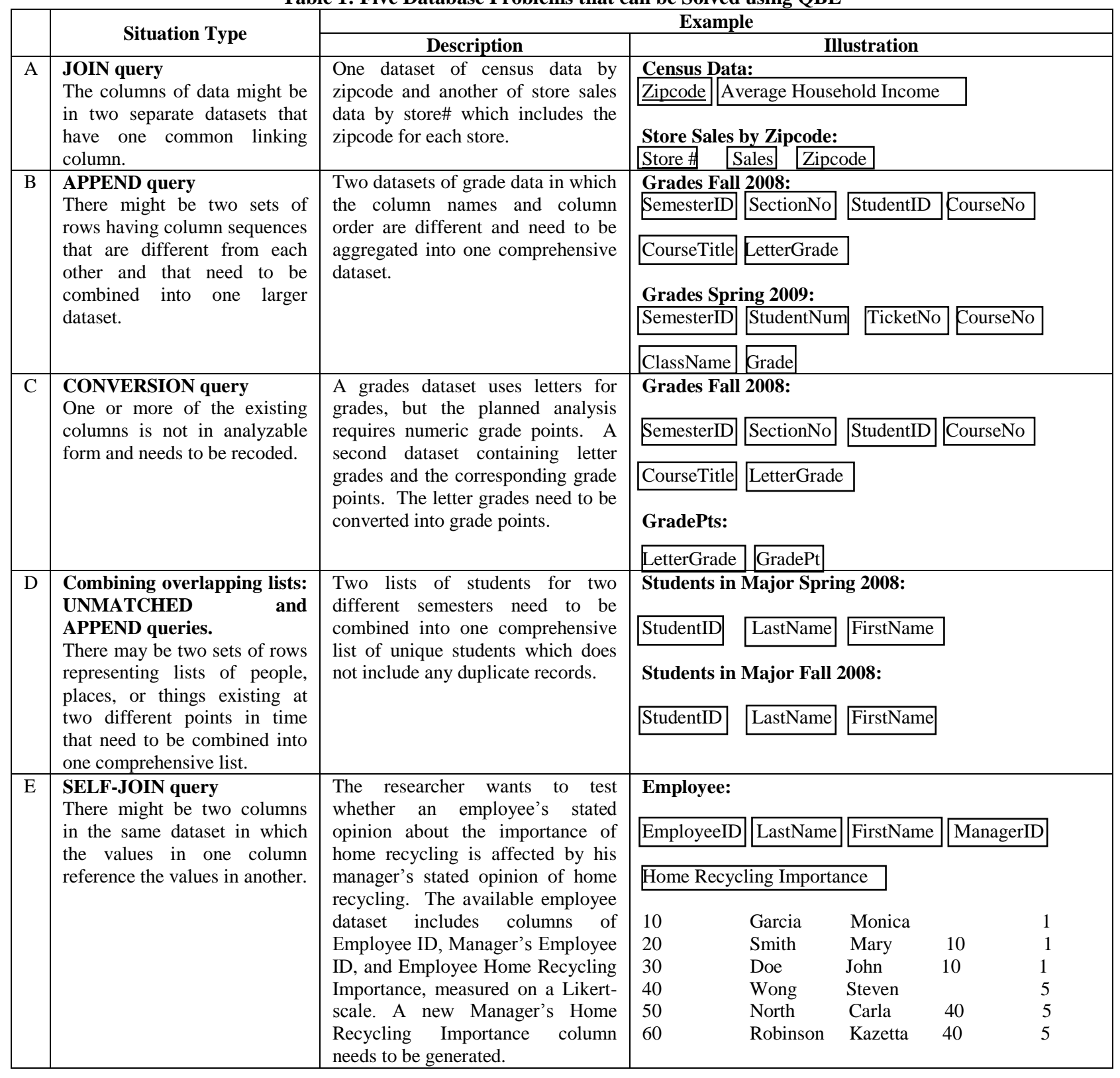


A. Combining the Columns from Two Related Tables (using a JOIN query).

1. Open the DataPrep101.mdb file. Be sure to turn off the security features (see Figures $3 \& 4$ ). The following tables will be used for this example: (1) 01_StoreSalesByZipCode and (2) 01_CensusData. Figures 13 and 14 show the datasets for these two tables. Notice that the two tables have a common column, zip code. The goal will be to have a one dataset with the columns of Store\#, Sales, Zip, and AvgHouseholdIncome.

Figure 13: 01_StoreSalesByZipCode Table

\begin{tabular}{|r|c|c|}
\hline \multicolumn{2}{|c|}{ 01_StoreSalesByZipcode } & \\
\hline Store\# & Sales & Zip \\
\hline & $\$ 10,500,000$ & 0000 \\
\hline 2 & $\$ 21,680,000$ & $\$ 0000$ \\
3 & $\$ 15,583,000$ & $\$ 0000$ \\
\hline 4 & $\$ 26,400,700$ & 0000 \\
\hline
\end{tabular}

Figure 14: 01_CensusData Table

\begin{tabular}{|c|r}
\hline Zipcode & AvgHouseholdIncome \\
20000 & $\$ 70,000$ \\
30000 & $\$ 80,000$
\end{tabular}

2. A JOIN query will be used to achieve this goal. A JOIN is a specific form of SELECT query that combines the data from two or more tables based upon common fields. Here's the process: Click the Create tab, then click on the Query Design button on the toolbar (see Figure 15). The Show Table dialog box appears (see Figure 16).

Figure 15: Creating a Query Using the Query Design Facility

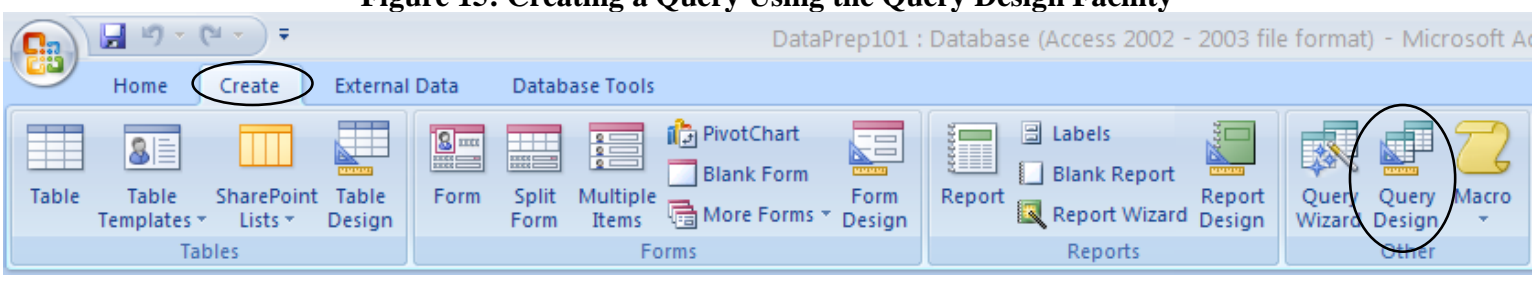

Figure 16: Show Table Dialog Box

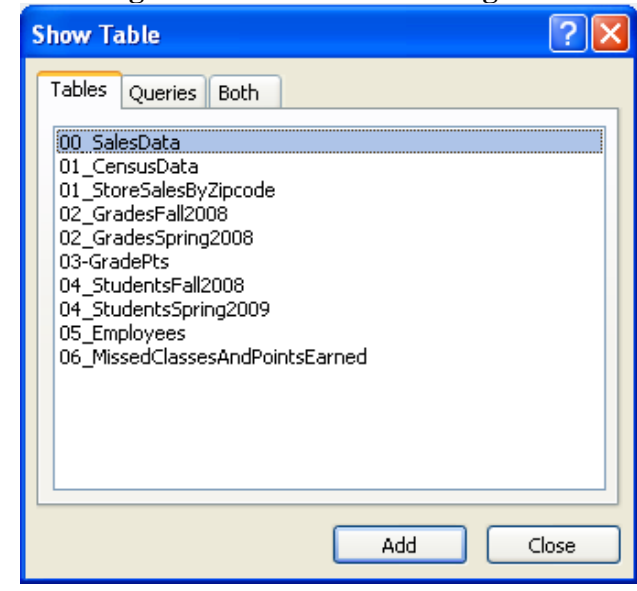


3. In the Show Table dialog box, select 01_StoreSalesByZipcode table; click Add. Next, select the 01_CensusData table; click Add. Click Close to close the dialog box. Your screen should now look like Figure 17.

4. The next step is to JOIN the two tables on the common field of zip code. This JOIN is imperative to the goal of creating one dataset. Click and drag the Zip field from the 01_StoreSalesByZipcode table to the Zipcode field of the 01_CensusData table. Your screen should now look like Figure 18.
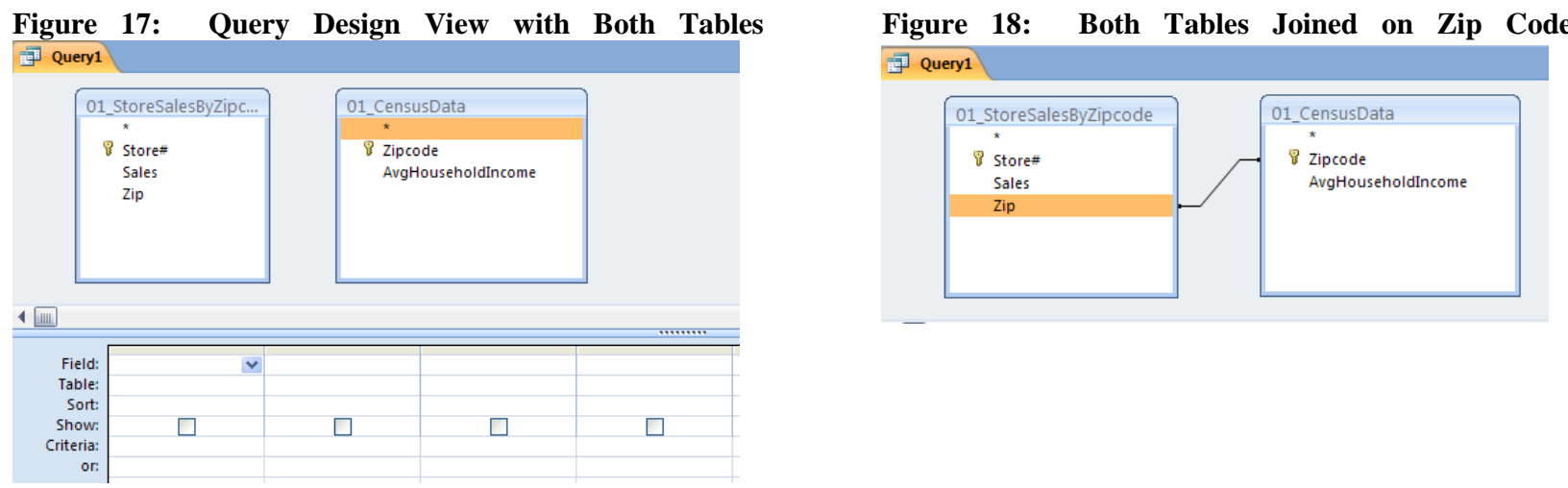

5. Now the fields from the tables need to be brought down to the field criteria grid. Double click the title bar of the 01_StoreSalesByZipcode table box. Click and drag the highlighted fields from the table box down to the field criteria grid (see Figure 19).

6. From the 01_CensusData table box, click and drag the AvgHouseholdIncome field to the field criteria grid. The Zipcode field does not need to be brought down, as it would only duplicate the zip codes in two columns. Your screen should now look like Figure 20.

Figure 19: Moving Fields to Field Criteria Grid

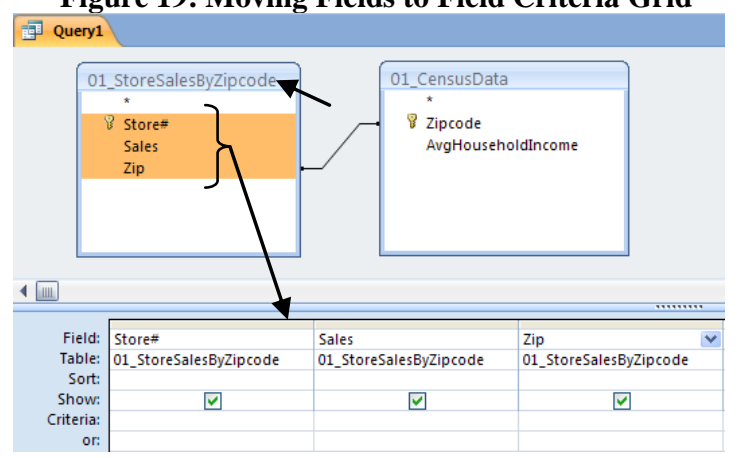

Figure 20: Moving Fields to Field Criteria Grid Part 2

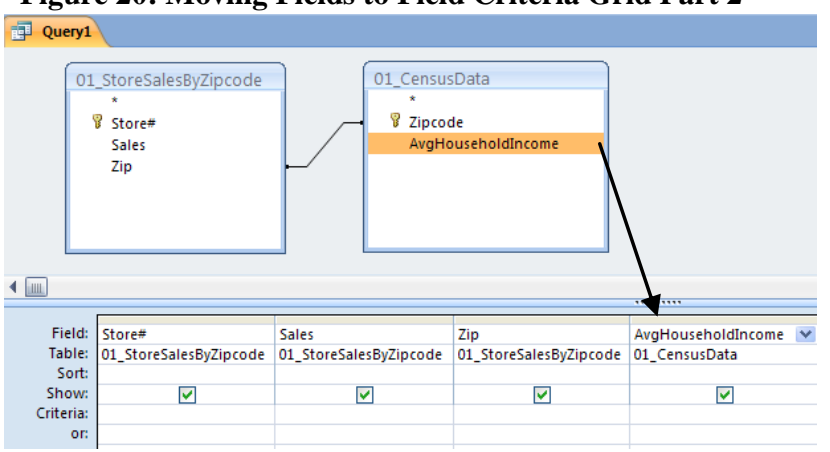

7. The query is ready to be run. To accomplish this, click the Run (!) button on the toolbar (see Figure 21). Figure 22 shows the query results with data from both of the tables. Phase III will demonstrate how to import this new dataset into SPSS. 
Figure 21: The Query Run Button

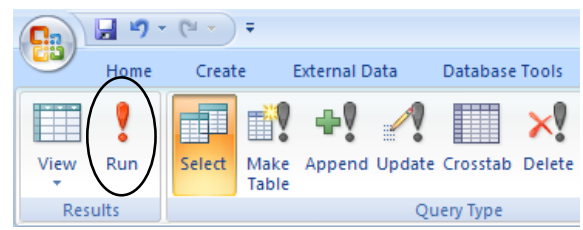

Figure 22: Query Results with Data from Both Tables

\begin{tabular}{|c|c|c|c|c|c|}
\hline \\
\hline \multicolumn{6}{|c|}{$\begin{array}{l}\text { Query1 } \\
\text { Store\# }\end{array}$} \\
\hline & & 1 & $\$ 10,500,000$ & 20000 & $\$ 70,000$ \\
\hline & & 3 & $\$ 15,583,000$ & 20000 & $\$ 70,000$ \\
\hline & & 2 & $\$ 21,680,000$ & 30000 & $\$ 80,000$ \\
\hline & & 4 & $\$ 26,400,700$ & 30000 & $\$ 80,000$ \\
\hline
\end{tabular}

8. Click the Save button (see Figure 23). The Save As dialog box appears. Name this new query: 01_Join. Click OK. The new query now shows up on the query list on the left-hand side of the screen (see Figure 24).

Figure 23: Query Save As Dialog Box

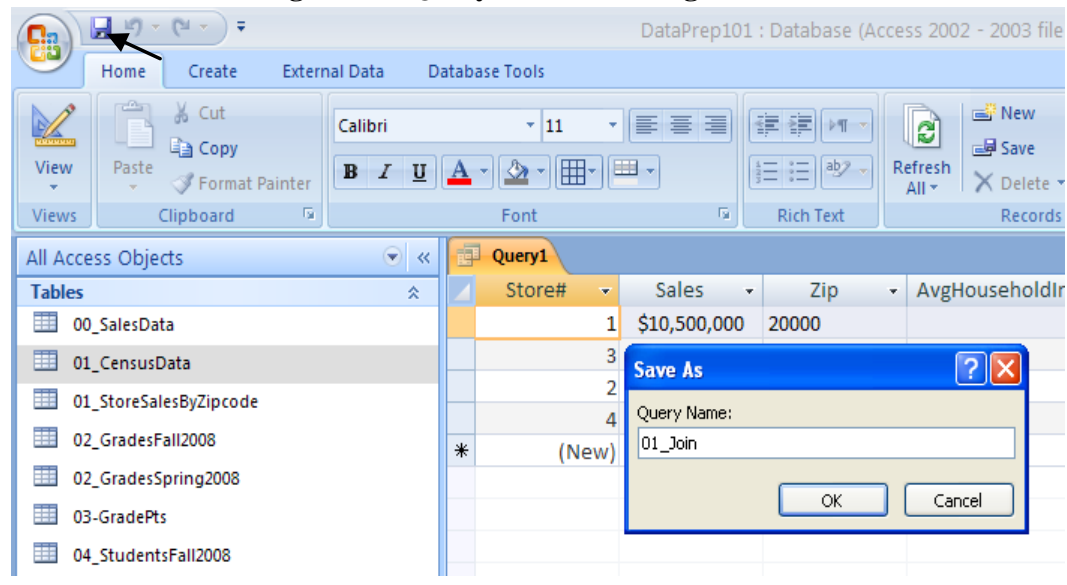

Figure 24: 01_Join in Query List

\begin{tabular}{|l|l|}
\hline All Access Objects & $\hat{\wedge}$ \\
\hline Tables & 00_SalesData \\
01_CensusData \\
01_StoreSalesByZipcode \\
02_GradesFall2008 \\
02_GradesSpring2008 \\
03_GradePts \\
04_StudentsFall2008 \\
04_StudentsSpring2009 \\
05_Employees \\
06_MissedClassesAndPointsEarned \\
\hline Queries \\
\hline 01_Join \\
\hline 06_MissedClassesAndPointsEarned_Query
\end{tabular}

9. Part $\mathrm{A}$ is now complete. The reader may now continue on to part $\mathrm{B}$ or close the DataPrep101.mdb file.

B. Adding the Rows in One Table into Another Table (using an APPEND query).

1. Open the DataPrep101.mdb file. Be sure to turn off the security features (see Figures $3 \& 4$ ). The following tables will be used for this example: (1) 02_GradesSpring2008 and (2) 02_GradesFall2008. Figures 25 and 26 show the datasets for these two tables. Notice that the two tables have all fields matching, however they are not in the same order, nor do all of the column headings match. The goal will be to have a one new table (named: 02_GradesAll) that includes all four records for 2008 .

Figure 25: 02_GradesSpring2008 Table
\begin{tabular}{|l|l|l|l|l|l|}
\hline 02_GradesSpring2008 & & & \\
\hline SemesterID - & SectionNo & StudentID & CourseNo & CourseTitle & LetterGrade - \\
\hline 20081 & 12456 & 384473999 & SOC100 & Principles of Sociology & B \\
\hline 20081 & 23500 & 245894533 & ACCT200 & Financial Accounting & C \\
\hline * & & & & & \\
\hline
\end{tabular}

Figure 26: 02_GradesFall2008 Table

\begin{tabular}{|c|c|c|c|c|c|c|c|}
\hline \multicolumn{8}{|c|}{ 02_GradesFall2008 } \\
\hline 4 & SemesterID - & StudentNum - & TicketNo & CourseNo - & ClassName & Grade & - \\
\hline & 20082 & 269475553 & 22679 & EDUC350 & Educational Psychology & B- & \\
\hline & 20082 & 684688392 & 45833 & MATH200 & College Algebra & $\mathrm{C}+$ & \\
\hline * & & & & & & & \\
\hline
\end{tabular}


2. Let's say that we want to be able to create the complete list of grades while leaving both of the original semester tables intact. We can accomplish this by first copying one of the two tables, say Spring, and then inserting duplicates of the fall records into the new table. Here's how it goes: The first step is to copy the 02_GradesSpring2008 table. On the left-hand side of the screen, under the Tables list, locate the 02_GradesSpring2008 table name (see Figure 27). Right click on it to make the shortcut menu appear. Choose Copy from the list.

3. Click the Paste button (see Figure 28), the Paste Table As dialog box appears. Name the new table: 02_GradesAll, then click OK. The 02_GradesAll table now shows up on the Tables list on the left-hand side of the screen. Remember, this table only includes the two records from the 02_GradesSpring2008 table. The next step is to add the two records from the 02_GradesFall2008.

Figure 27: Copy 02_GradesSpring2008 Table

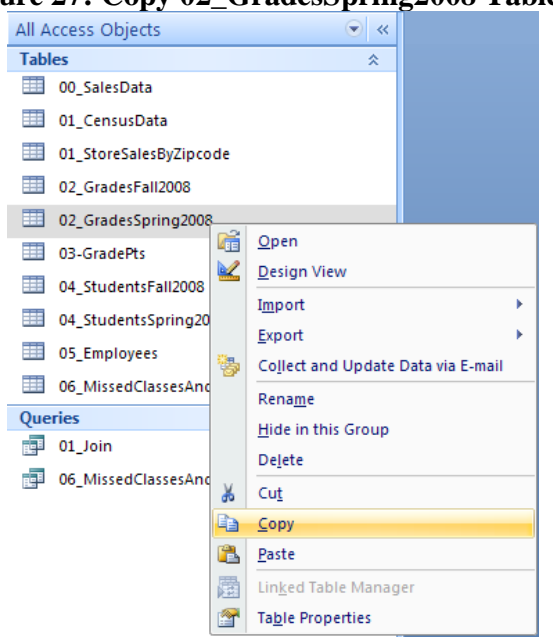

Figure 28: Paste Table As 02_GradesAll

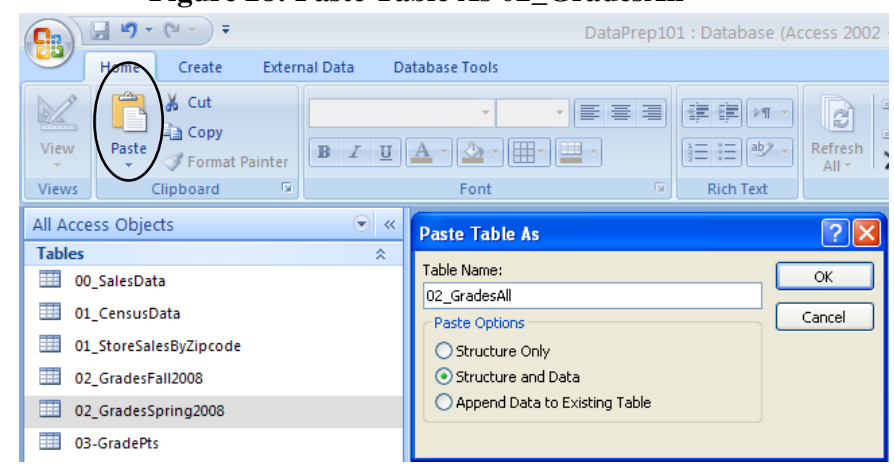

4. An APPEND query will be used to achieve this goal. Click the Create tab, then click on the Query Design button on the toolbar (see Figure 15). The Show Table dialog box appears, click Close. Click the Append button on the toolbar (see Figure 29). The Append dialog box appears. Choose the 02_GradesAll table as the file to append (or add records to), click $\mathbf{O K}$.

Figure 29: Append to 02_GradesAll Table

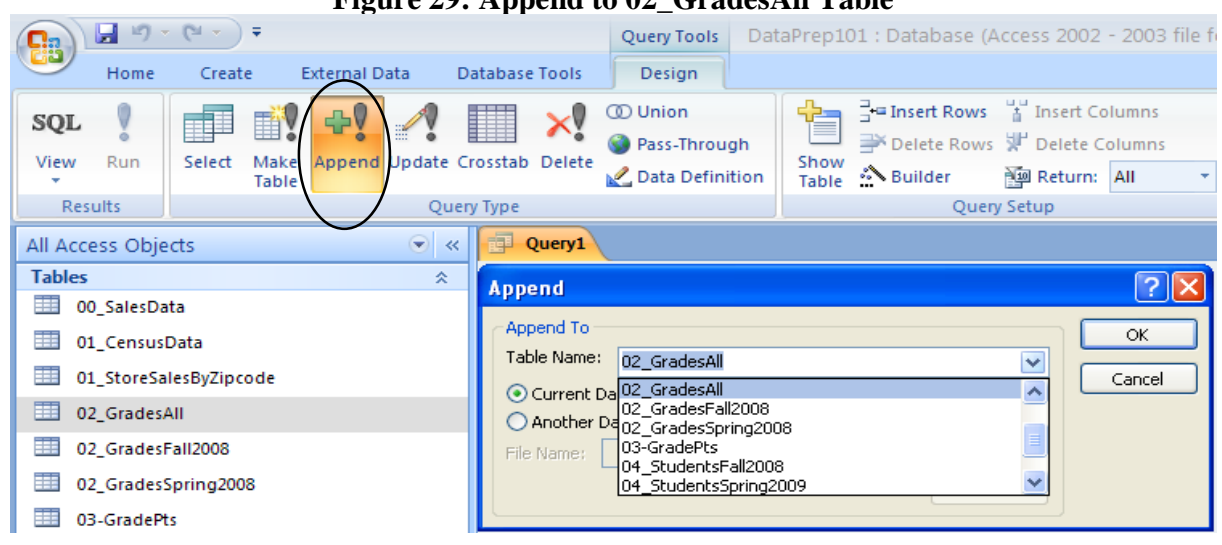

5. Click the Show Table button on the toolbar (see Figure 30); the Show Table dialog box appears. Highlight the 02_GradesFall2008 table from the list, click Add.

6. The next step is to add the fields from the 02_GradesFall2008 table box to the field criteria grid (see Figure 31). Double click the title bar of the 02_GradesFall2008 table box. Click and drag 
the highlighted fields from the table box down to the field criteria grid. Notice that only the SemesterID and CourseNo fields are currently matching up with the fields in the 02_GradesAll table because the fields were not named consistently for the two semesters. Now the remaining four fields in 02_GradesFall2008 need to be manually matched up with the corresponding field names in 02_GradesAll.

7. On the Append To row (see Figure 32), click the cell under StudentNum, click the Down Arrow and choose StudentID from the list. Continue with the three remaining field pair (TicketNo $=$ SectionNo, ClassName $=$ CourseTitle, and Grade $=$ LetterGrade $)$.

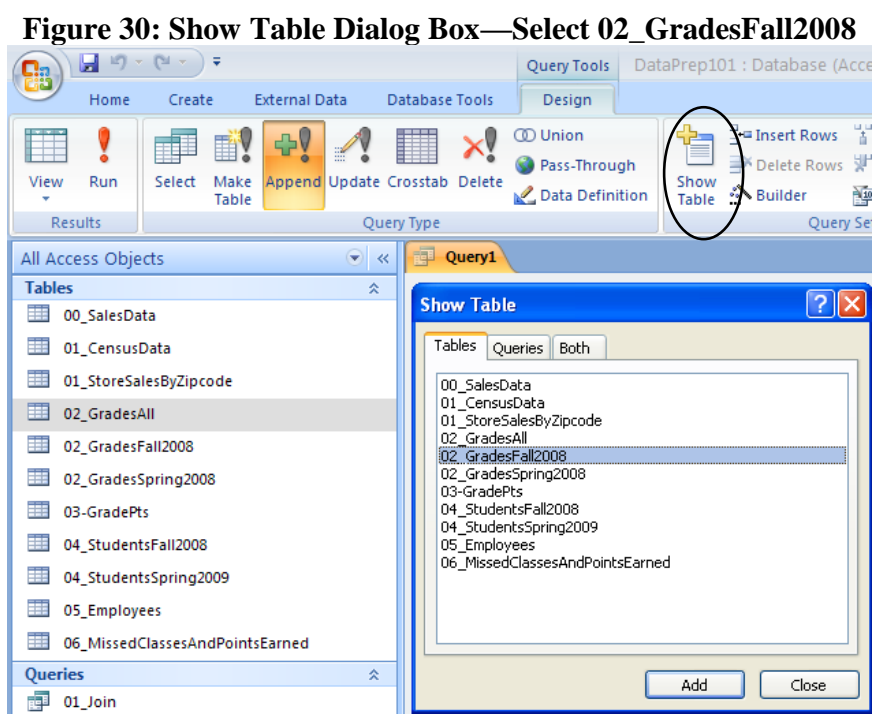

Figure 31: Bring All Fields Down to the Field Criteria Grid

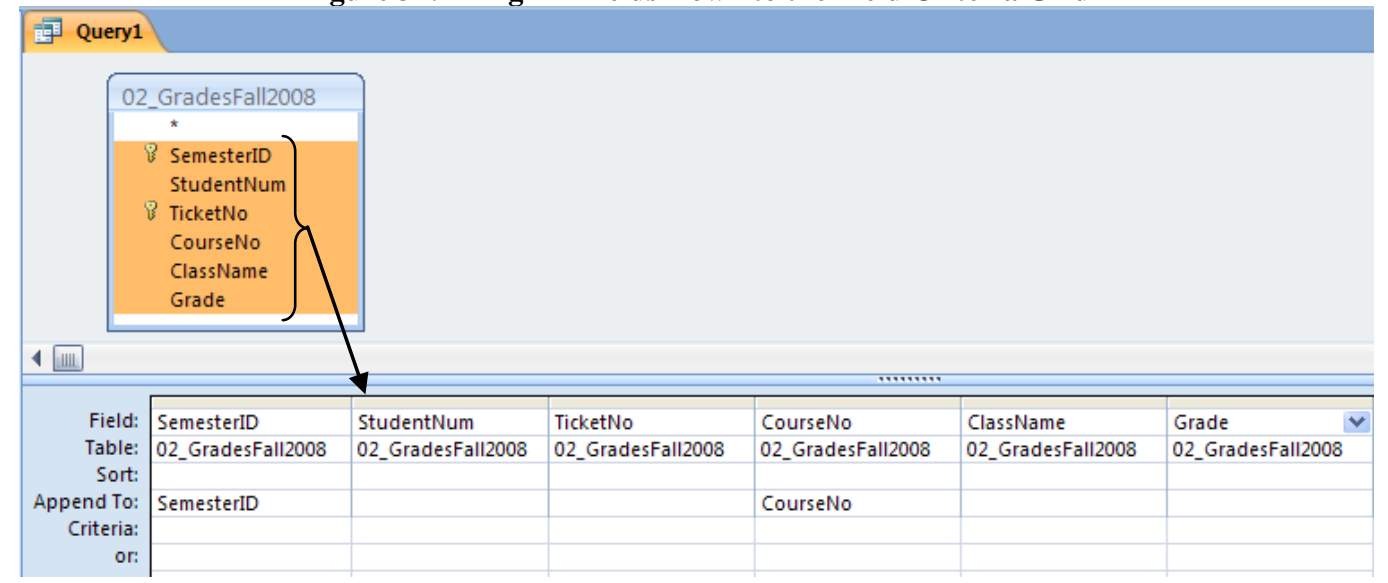




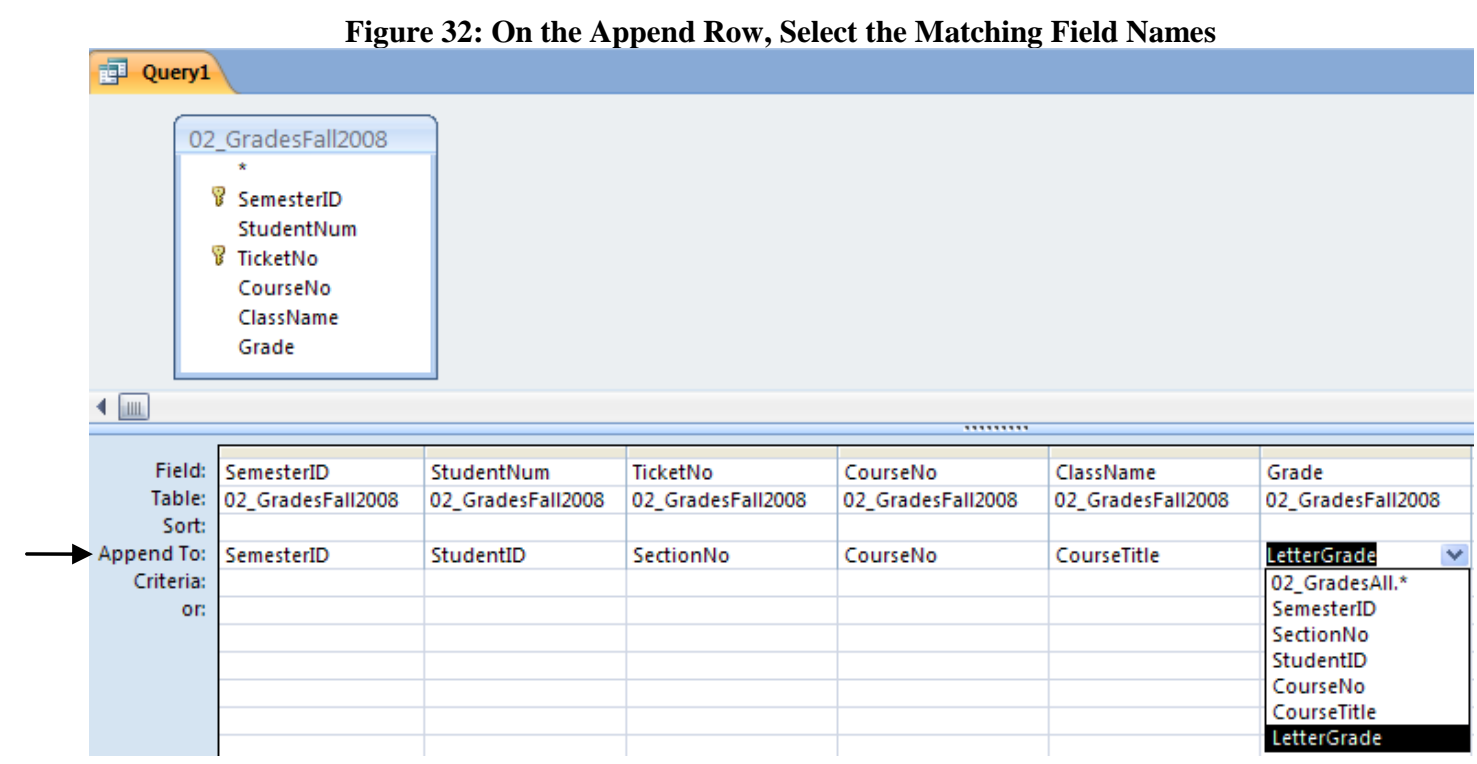

8. The final step is to run this APPEND query. Click Run (!). A dialog box appears warning that "You are about to append 2 rows (see Figure 33). Since this is what we want to do, click Yes.

9. Congratulations, you have successfully combined the two data sets. Click the Save button and save this query as 02_CombiningDataSets (see Figure 34). Click OK.

Figure 33: You Are About to Append 2 Row(s) Dialog Box

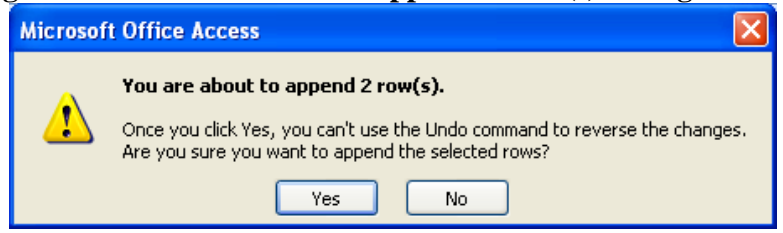

Figure 34: Save the Append Query

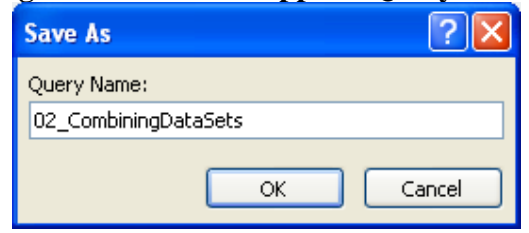

10. The 02_GradesAll table now includes all four grade records from Spring and Fall 2008 semesters (see Figure 35).

Figure 35: Appended 02_GradesAll Table

\begin{tabular}{|c|c|c|c|c|c|c|}
\hline \multicolumn{7}{|c|}{ 䎳 02_GradesAll } \\
\hline & SemesterID - & SectionNo & StudentID . & CourseNo & CourseTitle & LetterGrade . \\
\hline & 20081 & 12456 & 384473999 & SOC100 & Principles of Sociology & B \\
\hline & 20081 & 23500 & 245894533 & АССТ 200 & Financial Accounting & $\mathrm{C}$ \\
\hline & 20082 & 22679 & 269475553 & EDUC350 & Educational Psychology & B- \\
\hline & 20082 & 45833 & 684688392 & MATH2O0 & College Algebra & $\mathrm{C}+$ \\
\hline
\end{tabular}

11. Part $\mathrm{B}$ is now complete. The reader may now continue on to part $\mathrm{C}$ or close the DataPrep101.mdb file. 
C. Recoding a Column in One Table (using data stored in a second CONVERSION table).

1. Open the DataPrep101.mdb file. Be sure to turn off the security features (see Figures $3 \& 4$ ). The following tables will be used for this example: (1) 02_GradesFall2008 and (2) 03_GradePts. Figures 36 and 37 show the datasets for these two tables. Notice that the 02_GradesFall2008 table has a Grade column and the 03_GradePt table includes both a LetterGrade and a GradePt field. Our goal here will be to create one new dataset that contains all of the fields from the 02_GradesFall2008 table and also includes the GradePt column.

Figure 36: 02_GradesFall 2008 Table

\begin{tabular}{|c|c|c|c|c|c|c|}
\hline \multicolumn{7}{|c|}{ 02_GradesFalli2008 } \\
\hline 4 & SemesterID . & StudentNum - & TicketNo - & CourseNo . & ClassName & Grade \\
\hline & 20082 & 269475553 & 22679 & EDUC350 & Educational Psychology & B- \\
\hline & 20082 & 684688392 & 45833 & MATH2OO & College Algebra & C+ \\
\hline 米 & & & & & & \\
\hline
\end{tabular}

Figure 37: 03_GradePts Table

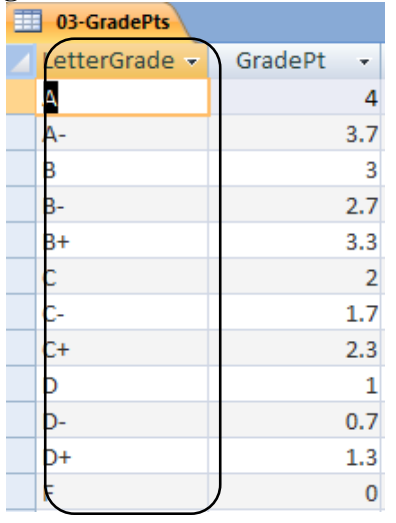

2. Click the Create tab, then click on the Query Design button on the toolbar (see Figure 15). The Show Table dialog box appears.

3. Choose the 02_GradesFall2008 table from the list; click Add (see Figure 38). Next, choose the 03_GradePts table from the list; Click Add. Click Close.

4. The next step is to JOIN the two tables on the common field of Grade/LetterGrade (see Figure 39). To do this, click and drag the Grade field from the 02_GradesFall2008 table to the LetterGrade field of the 03_GradePts table.

Figure 38: Add Tables to QBE

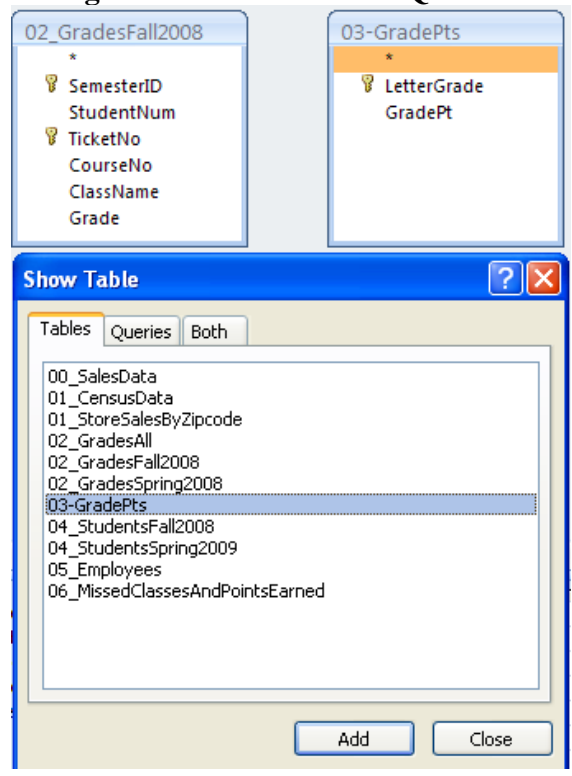

Figure 39: JOIN the Two Tables on Grade/LetterGrade Fields

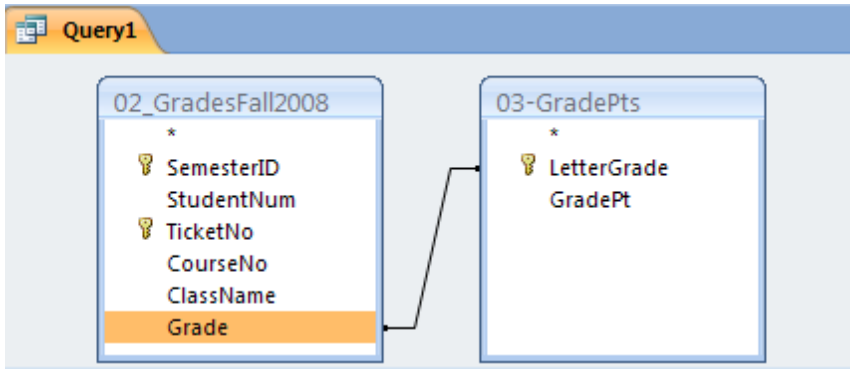


5. Now the fields from the tables need to be brought down to the field criteria grid. Double click the title bar of the 02_GradesFall2008 table box. Click and drag the highlighted fields from the table box down to the field criteria grid.

6. From the 03_GradePts table box, click and drag the GradePt field to the field criteria grid. The LetterGrade field does not need to be brought down, as it would only duplicate the Grade field from the other table. Your screen should now look like Figure 40.

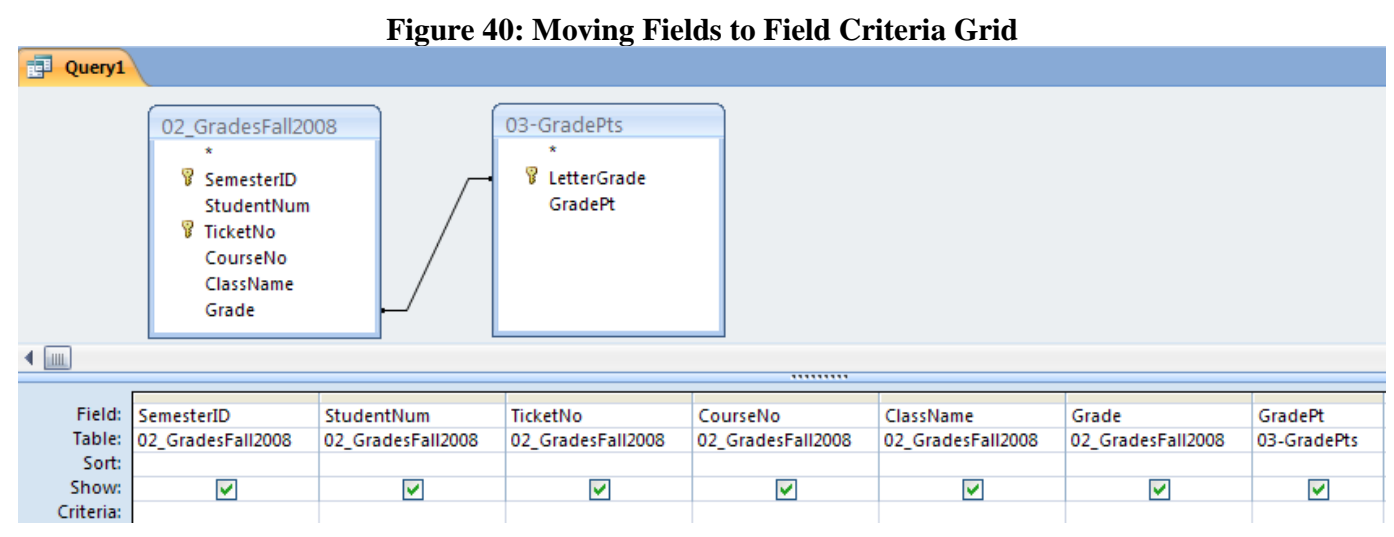

7. The final step is to run this query. Click Run (!). Congratulations, you have successfully used a CONVERSION table to combine the two datasets. The results of the query are in Figure 41. Click the Save button and save this query as 03_ConversionTable. Click OK.

\begin{tabular}{|c|c|c|c|c|c|c|c|c|c|}
\hline \multicolumn{10}{|c|}{ 03_ConversionTable } \\
\hline 4 & SemesterID . & StudentNum . & TicketNo & CourseNo - & ClassName & Grade & - & GradePt & - \\
\hline & 20082 & 269475553 & 22679 & EDUC350 & Educational Psychology & B- & & & 2.7 \\
\hline & 20082 & 684688392 & 45833 & MATH2OO & College Algebra & $\mathrm{C}+$ & & & 2.3 \\
\hline 米 & & & & & & & & & \\
\hline
\end{tabular}

8. Part $\mathrm{C}$ is now complete. The reader may now continue on to part $\mathrm{D}$ or close the DataPrep101.mdb file.

D. Dealing with Tables Having Overlapping Data (using UNMATCHED and APPEND queries).

1. Open the DataPrep101.mdb file. Be sure to turn off the security features (see Figures $3 \& 4$ ). The following tables will be used for this example: (1) 04_StudentsFall2008 and (2) 04_StudentsSpring2009. Figures 42 and 43 show the datasets for these two tables. Notice that the two tables have exactly the same fields, however some of the rows are overlapping (i.e., showing in both tables). The ultimate goal is to have one complete list of unique students in a table named 04_StudentsAll. 
Figure 42: 04_StudentsFall2008 Dataset

\begin{tabular}{|l|l|l|}
\hline \multicolumn{1}{|c|}{ 04_StudentsFall2008 } & \\
\hline $\mathbf{1 1 1 1 1 1 1 1}$ & One & John \\
\hline 22222222 & Two & Sierra \\
\hline 333333333 & Three & Troy \\
\hline 444444444 & Four & Montana \\
\hline 555555555 & Five & Stefan \\
\hline 666666666 & Six & Damian \\
\hline 777777777 & Seven & Donzie \\
\hline
\end{tabular}

Figure 43: 04_StudentsSpring2009 Dataset

\begin{tabular}{|l|l|l|}
\hline \multicolumn{1}{|c|}{$\begin{array}{l}\text { 04_StudentsSpring } 2009 \\
\text { StudentID }\end{array}$} & LastName & FirstName \\
\hline $\mathbf{3 3 3 3 3 3 3 3 3}$ & Three & Troy \\
\hline 444444444 & Four & Montana \\
\hline 555555555 & Five & Stefan \\
\hline 666666666 & Six & Damian \\
\hline 777777777 & Seven & Donzie \\
\hline 888888888 & Eight & Oriel \\
\hline 999999999 & Nine & Nancy \\
\hline
\end{tabular}

2. The first step is to copy the 04_StudentsFall2008 table. On the left-hand side of the screen, under the Tables list, locate the 04_StudentsFall2008 table name (see Figure 44). Right click on it to make the shortcut menu appear. Choose Copy from the list.

3. Click the Paste button (see Figure 45); the Paste Table As dialog box appears. Name the new table: 04_StudentsAll, then click OK. The 04_StudentsAll table now shows up on the Tables list on the left-hand side of the screen. Remember, so far this new table only includes the records from the 04_StudentsFall2008 table. The next step is to find the unmatched records from the 04_StudentsSpring2009 table (LastNames Eight and Nine).

Figure 44: Copy 04_StudentsFall2008 Table

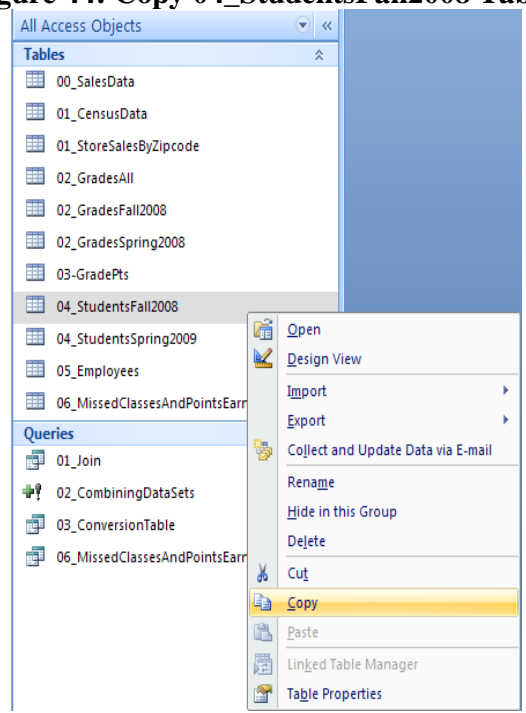

Figure 45: Paste Table As 04_StudentsAll

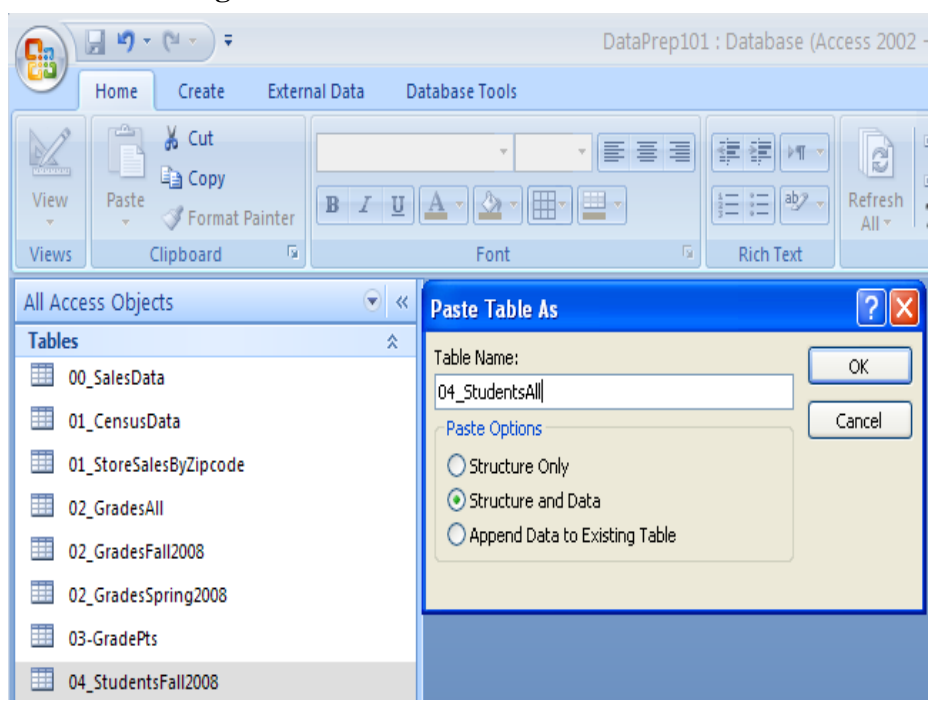

4. Click the Create button then click the Query Wizard button, the New Query dialog box appears (see Figure 46). Select Find Unmatched Query Wizard from the list; click OK. The next step is to choose the table with the new data (in this case 04_StudentsSpring2009).

5. In the first dialog box of the Find Unmatched Query Wizard, select the 04_StudentsSpring2009 table from the list, click Next (see Figure 47). The next step is to choose the table that contains the related records (in this case, 04_StudentsAll). 


\section{Figure 46: Launch the Find Unmatched Query Wizard}

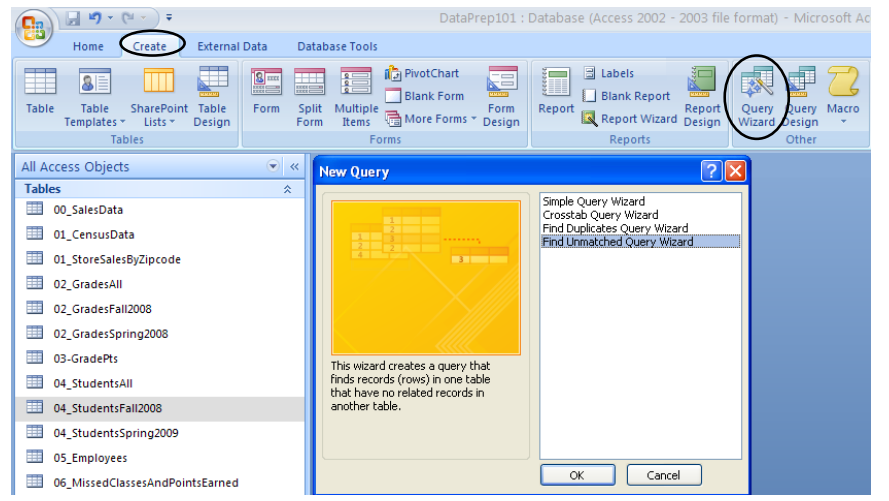

Figure 47: Unmatched Query Wizard-Screen 1

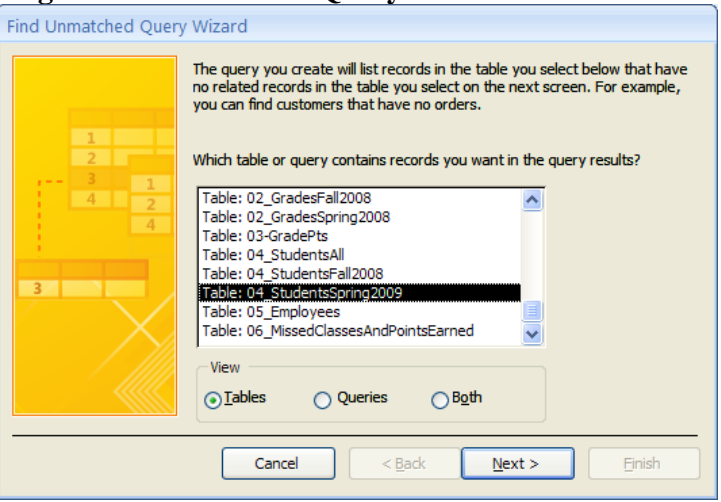

6. In the second dialog box, select the 04_StudentsAll table from the list; click Next (see Figure 48). The next step is to identify which fields are matching in both tables.

7. In the third dialog box, make sure that the StudentID field is selected on both sides (indicating that the records in the two tables should be compared on the basis of this key field); click Next (see Figure 49). The next step is to identify which fields should show in the query results.

Figure 48: Unmatched Query Wizard-Screen 2

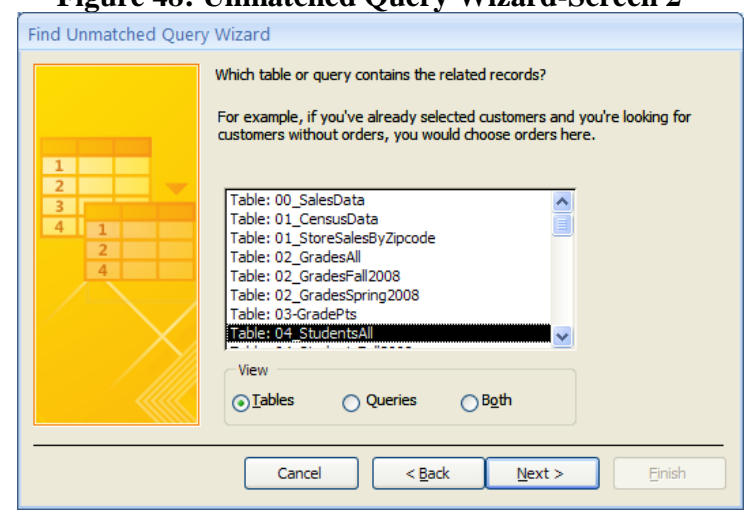

Figure 49: Unmatched Query Wizard-Screen 3

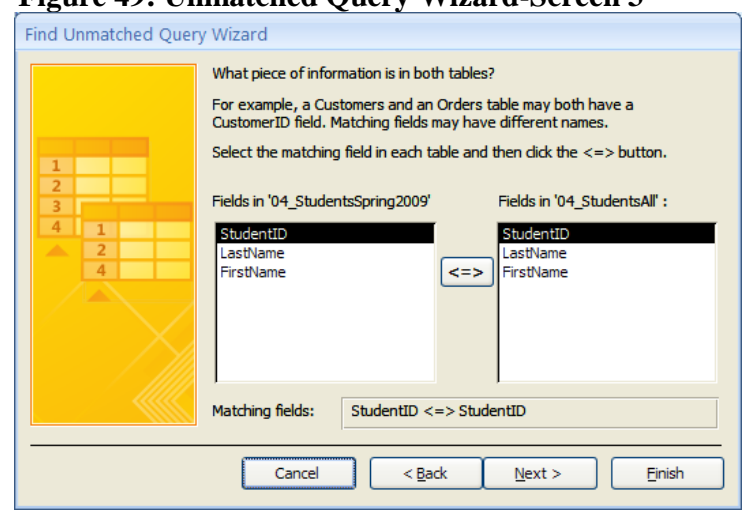

8. In the fourth dialog box, move all three fields (StudentID, LastName, and FirstName), from the left to the right, by clicking the >> button; click Next (see Figure 50).

9. This query must be named. In the next dialog box, type in 04_Spring2009NewStudents; click Finish (see Figure 51). The query results are now showing on the screen (see Figure 52). Notice that only the unmatched records (i.e., the new students for Spring 2009) are in the list.

Figure 50: Unmatched Query Wizard-Screen 4

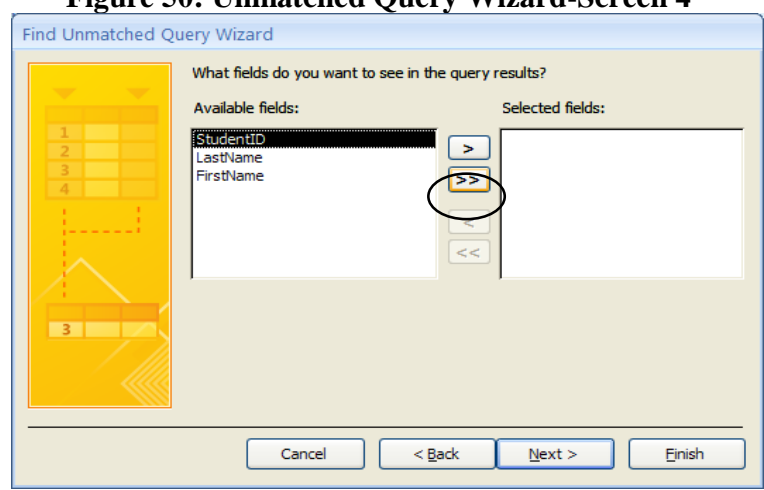

Figure 51: Name Query 04_Spring2009NewStudents

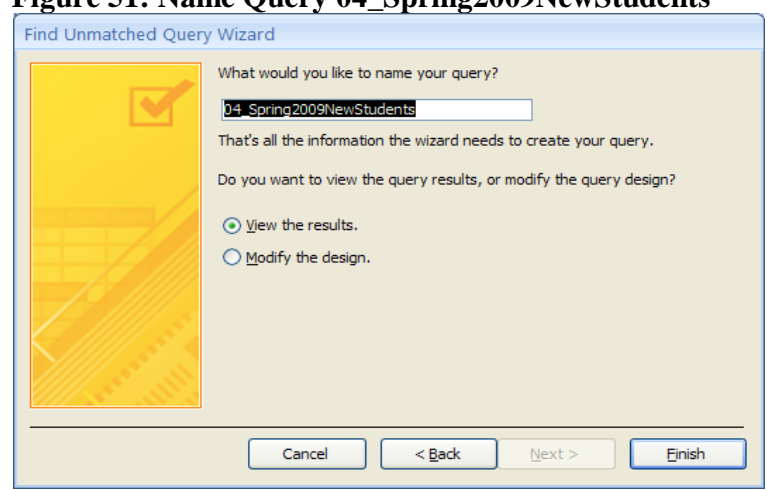


Figure 52: 04_Spring2009NewStudents Query Results

\begin{tabular}{|l|l|l|}
\hline $\begin{array}{l}\text { 04_Spring2009NewStudents } \\
\text { StudentID }\end{array}$ & LastName & FirstName \\
\hline 888888888 & Eight & Oriel \\
\hline 999999999 & Nine & Nancy \\
\hline
\end{tabular}

12. Now that the unmatched records have been identified, the next step is to APPEND (or add) these two records to the 04_StudentsAll table. Click the Create tab, then click on the Query Design button on the toolbar (see Figure 15). The Show Table dialog box appears, click Close. Click the Append button on the toolbar (see Figure 29). The Append Query dialog box appears. Choose the 04_StudentsAll table as the file to append (or add records to); click OK (see Figure 53).

13. Click the Show Table button on the toolbar. The Show Table dialog box appears. Click on the Queries tab and select the 04_Spring2009NewStudents query from the list (see Figure 54). Click Add. Click Close. Your screen should now look similar to Figure 55.

Figure 53: Append Query Dialog Box

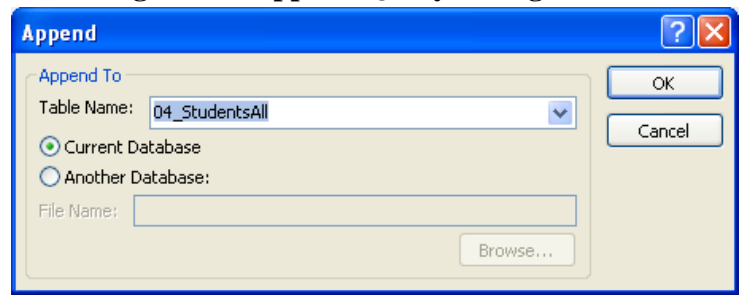

Figure 54: Show Table Dialog Box

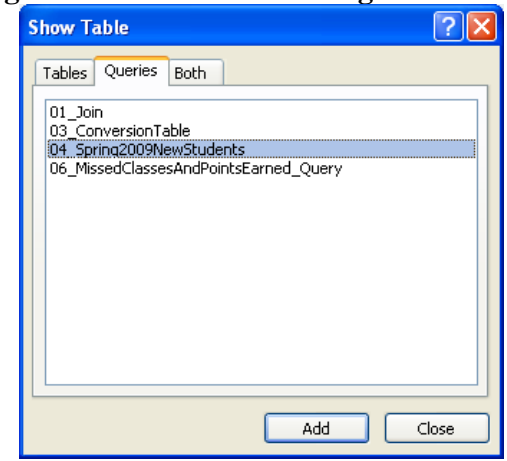

Figure 55: Append Query

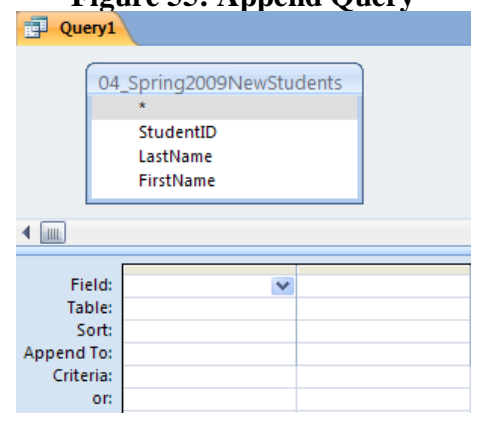

14. The next step is to add the fields from the 04_Spring2009NewStudents query box at the top of the screen to the field criteria grid at the bottom (see Figure 56). Double click the title bar of the 04_Spring2009NewStudents query box. Click and drag the highlighted fields from the query box down to the field criteria grid.

15. The next step would normally be to select the matching fields on the Append To row. Since the three field names are identical in the 04_Spring2009NewStudents query and the 04_StudentsAll table, they already match.

16. The final step is to run this APPEND query. Click Run (!). A dialog box appears warning that "You are about to append 2 rows." Click Yes.

17. Congratulations, you have successfully combined the two data sets. Click the Save button and save this query as 04_2008And2009Students. Click OK. 
18. If you view the 04_StudentsAll table, it will now contain the list of nine unique students from both semesters (See Figure 57).

Figure 56: Bring All Fields Down to the Field Criteria Grid

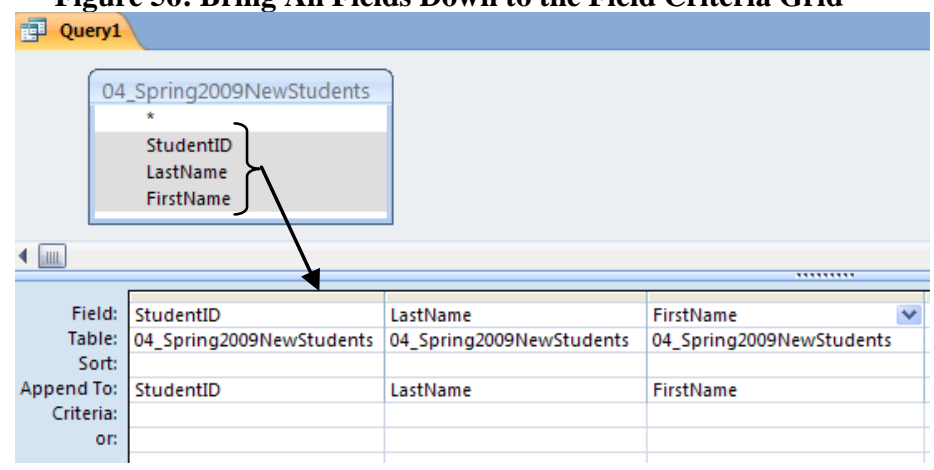

Figure 57: 04_StudentsAll Dataset

\begin{tabular}{|l|l|l|}
\hline $\begin{array}{l}\text { 04_StudentsAll } \\
\text { StudentID }\end{array}$ & & LastName \\
\hline 111111111 & One & FirstName \\
\hline 22222222 & John \\
\hline 333333333 & Three & Sierra \\
\hline 444444444 & Four & Troy \\
\hline 555555555 & Five & Stefantana \\
\hline 666666666 & Six & Damian \\
\hline 777777777 & Seven & Donzie \\
\hline 888888888 & Eight & Oriel \\
\hline 999999999 & Nine & Nancy \\
\hline
\end{tabular}

19. Part D is now complete. The reader may now continue on to part $\mathrm{E}$ or close the DataPrep101.mdb file.

E. $\quad$ Allowing the Matching of Values in One Column that Reference the Values of Another Column in the Same Table (using a SELF-JOIN query).

1. Open the DataPrep101.mdb file. Be sure to turn off the security features (see Figures $3 \& 4$ ). The following table will be used for this example: 05_Employees (see Figure 58). Notice that the ManagerID is actually the EmployeeID of the manager. The statistical goal is to see if the employee's opinion of HomeRecyclingImportance is influenced by (i.e., correlated with) the opinion of their manager. To this requires a dataset that contains the columns: EmployeeID, EmpOpinion, ManagerID, and MgrOpinion. Of course, the reader will correctly note that the 05_Employees table only contains the HomeReyclingImportance opinion of the Employee, not the Manager. But, since managers are, themselves employees, their opinions on recycling are also stored in the table. For example, Mary Smith's (Employee \#20) Rating is 2 and her manager's (Monica Garcia, Manager \#10) rating is 1 . The process for extracting data from a table in this way involves making a virtual copy of the original 05_Employees table and then linking the copy to the original and is called a SELF JOIN.

Figure 58: 05_Employees Table Dataset

\begin{tabular}{|c|c|c|c|c|c|c|c|c|c|c|}
\hline & EmployeelD & 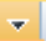 & LastName & 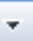 & FirstName & 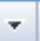 & ManagerID . &. & HomeRecyclinglmportance &. \\
\hline & & 10 & Garcia & & Monica & & & & & 1 \\
\hline & & 20 & Smith & & Mary & & & 10 & & 2 \\
\hline & & 30 & Doe & & John & & & 10 & & 1 \\
\hline & & 40 & Wong & & Steven & & & & & 5 \\
\hline & & 50 & North & & Carla & & & 40 & & 4 \\
\hline & & 60 & Robinson & & Kazetta & & & 40 & & 5 \\
\hline
\end{tabular}

2. To start, click the Create tab, then click on the Query Design button on the toolbar (see Figure 15). The Show Table dialog box appears. Select the 05_Employees table from the list. Click Add, then click Add again. You should now have two 05_Employees table boxes showing (see Figure 59). 
3. The next step is to JOIN the two copies of the table on the common field. This JOIN is unique, in that ManagerID and EmployeeID will be the common fields, therefore allowing us to create a SELF JOIN. Click and drag the ManagerID field from the 05_Employees table box to the EmployeeID field of the 05_Employees_1 table box. Your screen should now look like Figure 60 .

Figure 59: Two 05_Employee Table Boxes

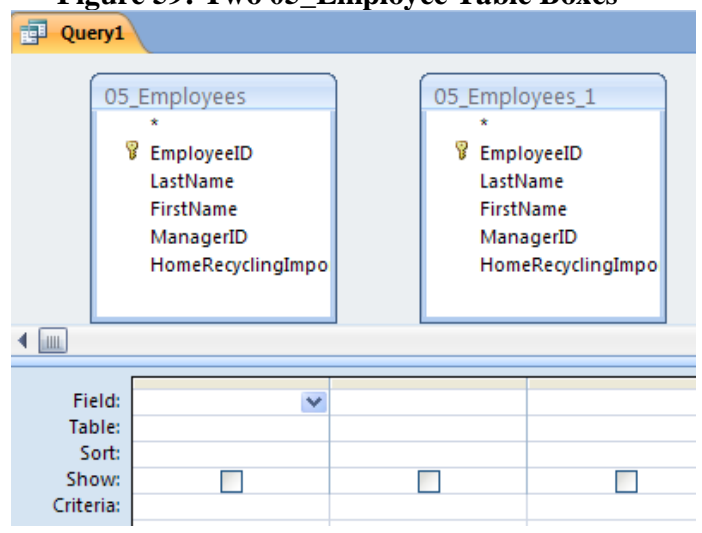

Figure 60: Join ManagerID to EmployeeID

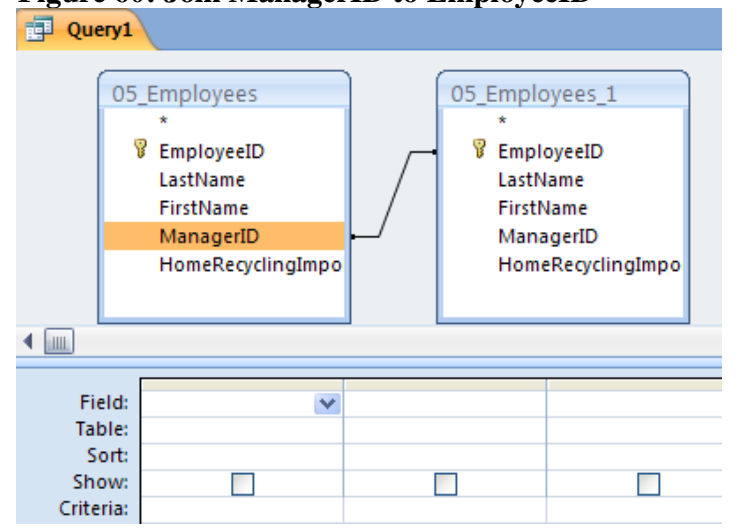

4. The next step is to bring down the required fields to the field criteria grid (see Figure 61). From the 05_Employees table box double-click the EmployeeID, then double-click the HomeRecyclingImportance. Next, from the 05_Employees_1 table box double-click the EmployeeID, then double-click the HomeRecyclingImportance.

5. The next step is to rename the two HomeRecyclingImportance fields to reflect the identity of the opinion and to rename the second EmployeeID field to ManagerID (see Figure 62). Type in EmpOpinion: in front of the first HomeRecyclingImportance field. Type in ManagerID: in front of the second EmployeeID. Finally, type in MgrOpinion: in front of the second HomeRecyclingImportance field.

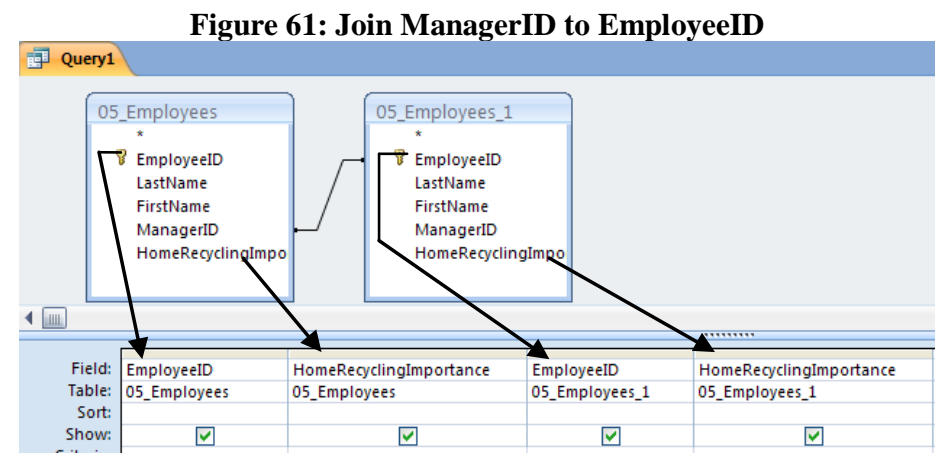

Figure 62: Renaming of the Fields

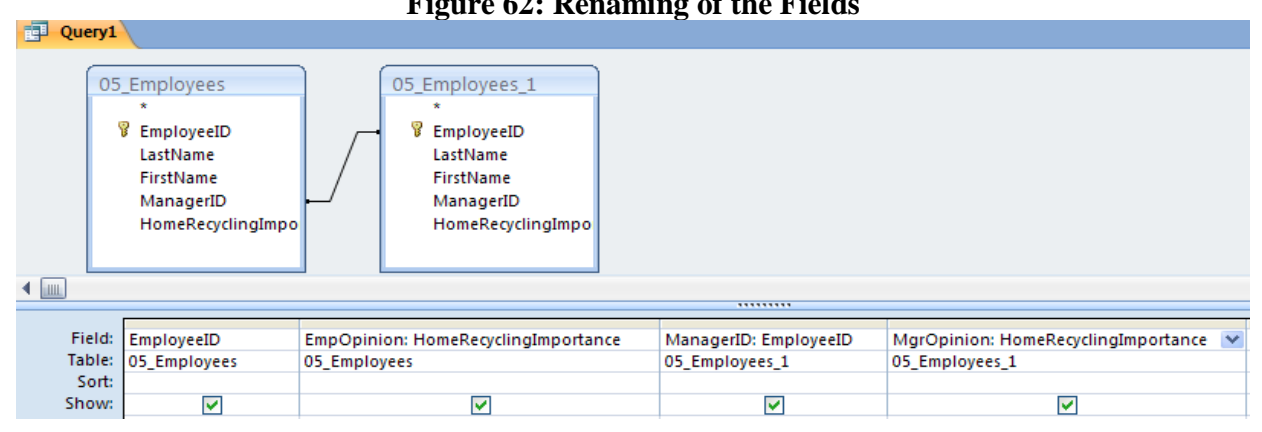


6. The final step is to run this query. Click Run (!). Congratulations, you have successfully used a SELF JOIN by using two copies of the same table. The results of the query are in Figure 63. It appears that the opinion of the manager does impact the opinion of the employee. Click the Save button and save this query as 05_EmployeeAndManagerOpinions. Click OK.

Figure 63: 05_EmployeeAndManagerOpinions Query Results

\begin{tabular}{|r|r|r|r|r|}
\hline 05_EmployeeAndManagerOpinions & & & MgrOpinion & - \\
\hline Employee - & EmpOpinion & - & ManagerID * & 1 \\
\hline 20 & 2 & 10 & 1 \\
\hline 30 & 1 & 10 & 5 \\
\hline 60 & 4 & 40 & 5 \\
\hline
\end{tabular}

7. Phase II is now complete. The reader may now continue on to Phase III or close the DataPrep101.mdb file.

\section{PHASE III: IMPORTING A RECONFIGURED DATASET FROM MS ACCESS INTO SPSS.}

Now that the five common ways of reconfiguring datasets using QBE have been covered, this third and final phase of the paper explains how to bring a reconfigured dataset from MS Access into SPSS for statistical analysis. Specifically, the reader will be importing the 06_MissedClassesAndPointsEarned_Query dataset from MS Access into SPSS and run a regression on this data. Although SPSS will be used in this example, many other statistical analysis packages (including MS Excel, SAS, or Minitab) could also be used.

A. The following steps will be used in importing the 06_MissedClassesAndPointsEarned_Query from MS Access into SPSS. Figure 64 shows the query results in MS Access.

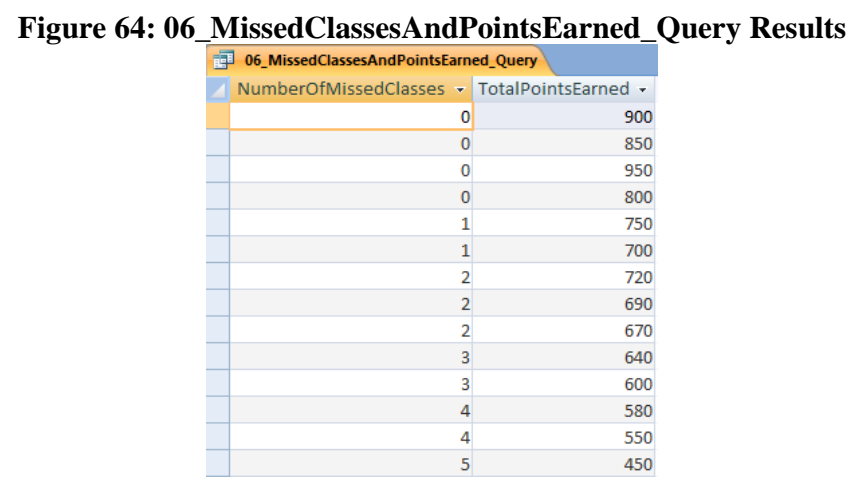

1. Launch SPSS so that a new data editor window appears (see Figure 65).

2. Before the query may be imported, a "Microsoft Data Source" must first be established. This is done by the use of SPSS's Database Wizard. To launch the wizard, from the menu select File/Open Database/New Query (see Figure 66). 
Figure 65: New SPSS Data Editor Window

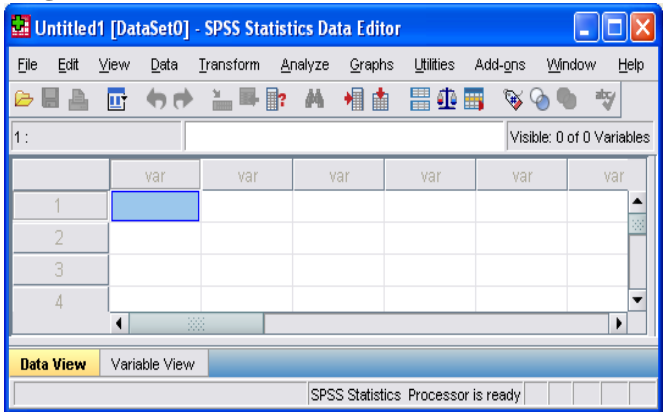

Figure 66: Accessing the Database Wizard

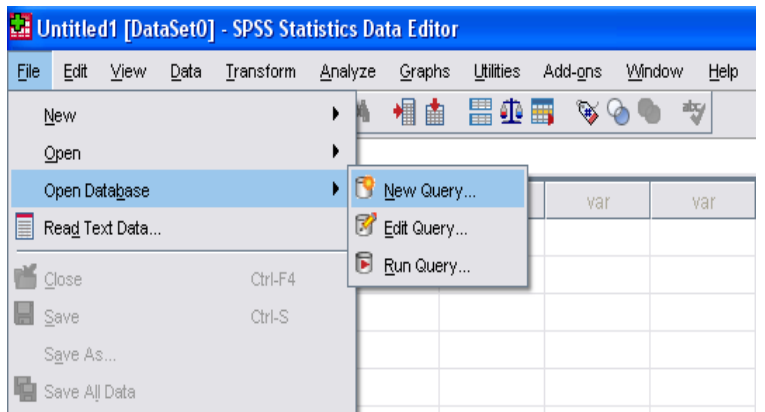

3. The Database Wizard appears. Select MS Access Database from the list; click Next (see Figure 67).

4. The ODBC Driver Login window appears. The next step is to locate the DataPrep101.mdb file. Click the Browse button; locate the file; click OK (see Figure 68).

Figure 67: Database Wizard

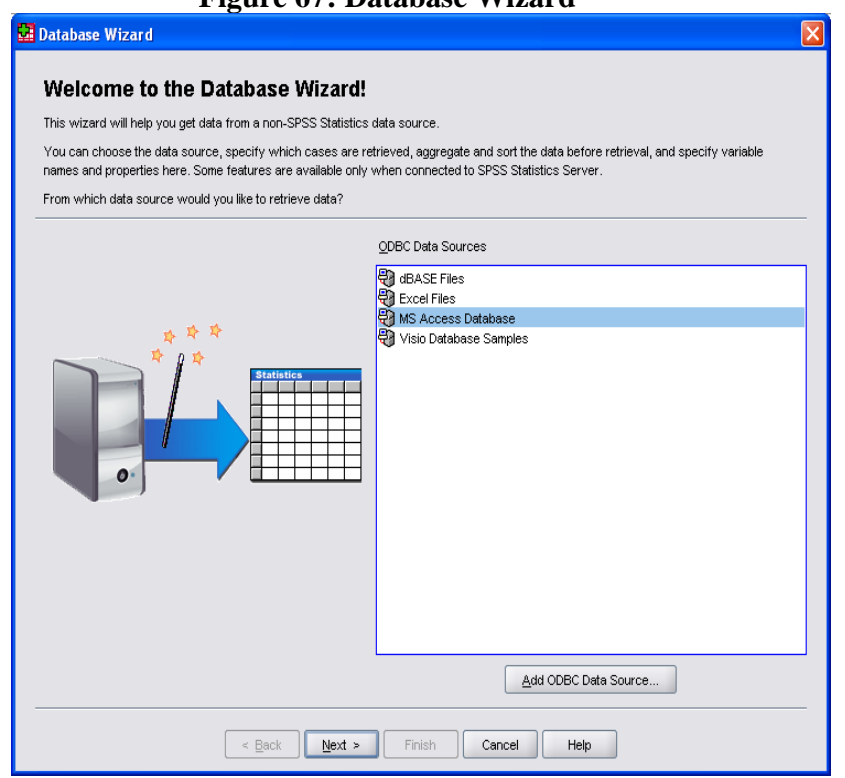

Figure 68: Selecting the Database

Fat ODBC Driver Login
Data Source: MS Access Database
Database:

5. The Database Wizard-Select Data window appears (see Figure 69). Notice that all of the tables are showing on the left-hand side of the window, however, none of the queries are visible. Click the Checkbox next to Views and the queries are also included in the list. (see Figure 70).

6. Select the 06_MissedClassesAndPointsEarned_Query from the list, click the $\uplus$ to move the two fields from the query to the right-hand side of the screen. Your screen should now look like Figure 71. 
Figure 69: Database Wizard-Select Data-Screen 1

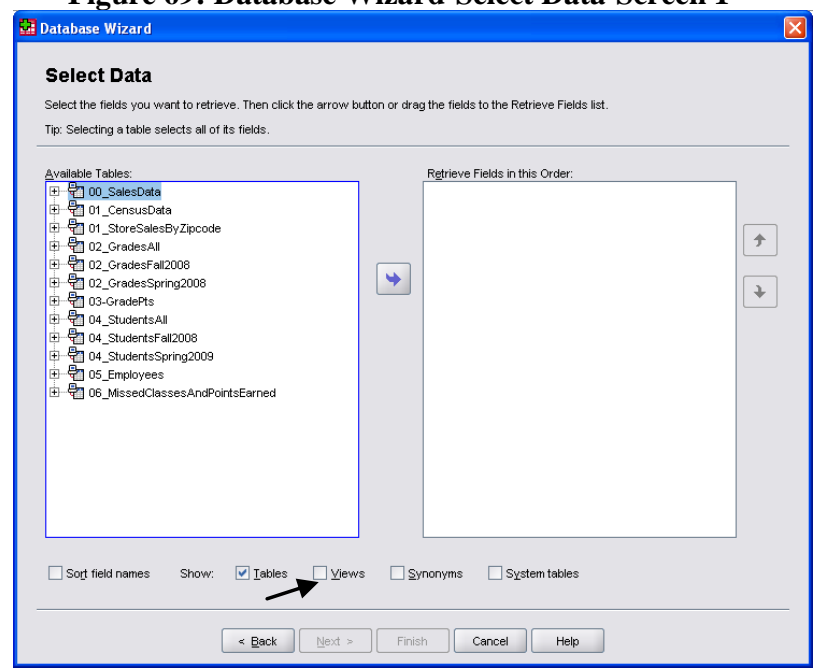

Figure 70: Database Wizard-Select Data-Screen 2

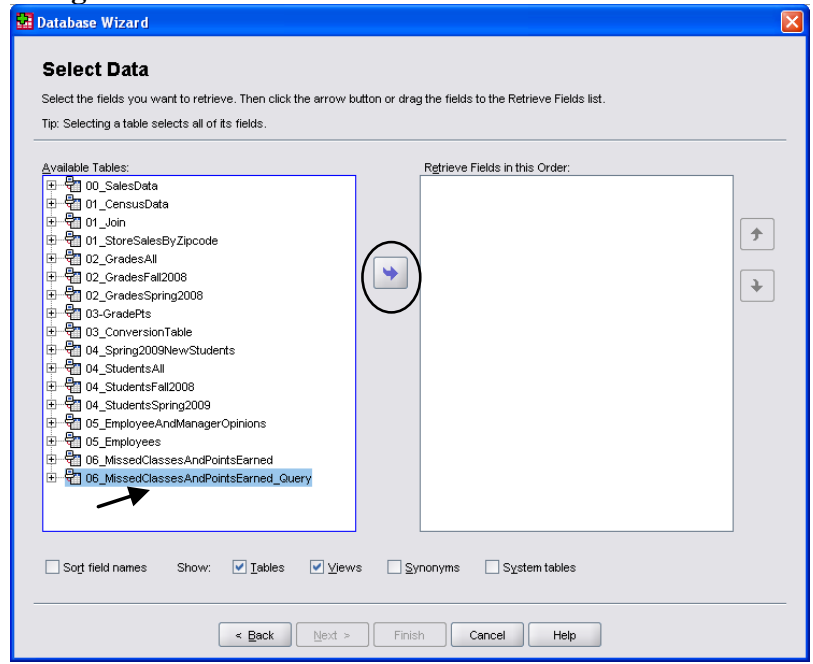

7. Notice that both the NumberOfMissingClasses and the TotalPointsEarned fields are now listed. Click Finish. The data is now showing in the SPSS Data Editor (see Figure 72). Congratulations, you have successfully imported the dataset into SPSS. Follow these same steps to bring in any table or query from an MS Access database.

Figure 71: Database Wizard-Select Data-Screen 3

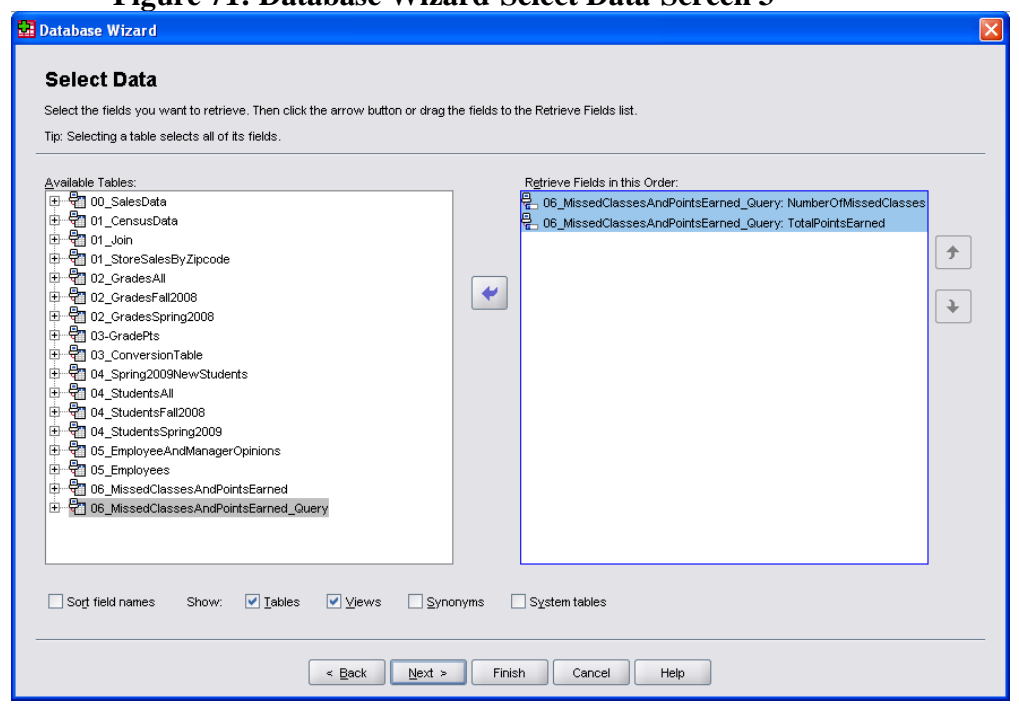

Figure 72: Data in SPSS Data Editor

\begin{tabular}{|c|c|c|c|}
\hline \multicolumn{4}{|c|}{ Hititled2 [DataSet1] - SPSS Statistics Data Editor } \\
\hline \multirow{2}{*}{\multicolumn{4}{|c|}{ 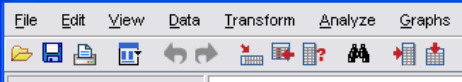 }} \\
\hline & & & \\
\hline \multicolumn{4}{|c|}{$1:$ NumberOfMissedClasses 0.0} \\
\hline & $\begin{array}{c}\text { NumberOfMissed } \\
\text { Classes }\end{array}$ & TotalPointsEarr & ined \\
\hline 1 & 0 & & 900 \\
\hline 2 & 0 & & 850 \\
\hline 3 & 0 & & 950 \\
\hline 4 & 0 & & 800 \\
\hline 5 & 1 & 7 & 750 \\
\hline 6 & 1 & 7 & 700 \\
\hline 7 & 2 & 7 & 720 \\
\hline 8 & 2 & E & 690 \\
\hline 9 & 2 & E & 670 \\
\hline 10 & 3 & B & 640 \\
\hline 11 & 3 & b & 600 \\
\hline 12 & 4 & 5 & 580 \\
\hline 13 & 4 & 5 & 550 \\
\hline 14 & 5 & 4 & 450 \\
\hline
\end{tabular}

B. The next step is to run the regression in SPSS. In this dataset the TotalPointsEarned is the dependent variable and the NumberOfMissingClasses is the independent variable. Follow these steps to run the regression.

1. From the menu, select Analyze/Regression/Linear (see Figure 73). The Linear Regression Dialog Box Appears (see Figure 74). 
2. On the right-hand side of the screen, select TotalPointsEarned and click the $\uplus$ next to the

Dependent: variable. Next, select the NumberOfMissingClasses and click the $\uplus$ next to the Independents(s): variable. Your screen should look similar to Figure 74.

Figure 73: Launch the Linear Regression

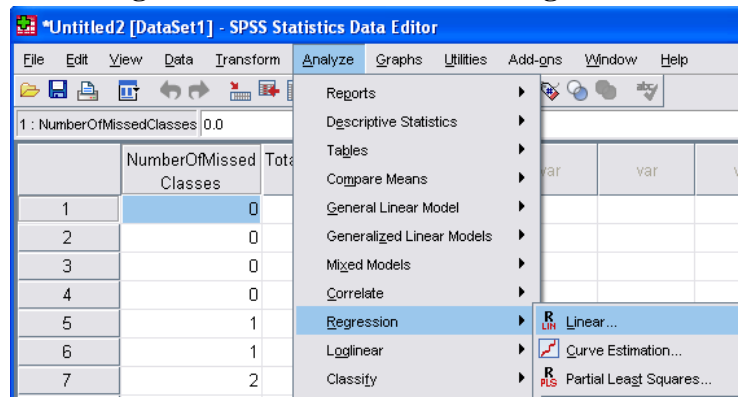

Figure 74: Linear Regression Dialog Box

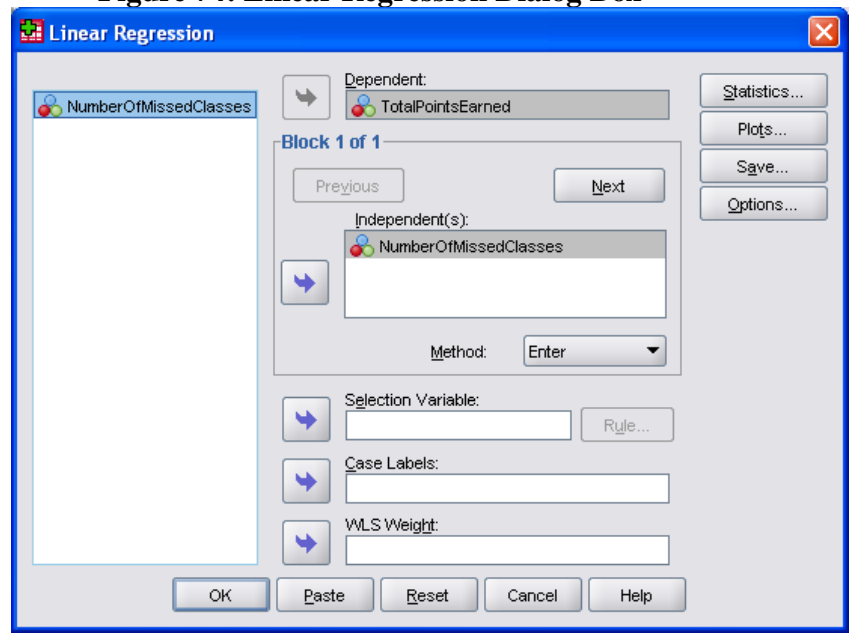

3. The regression is now ready to be run. Click OK. The results of the regression are now displayed in the SPSS Statistics Viewer (see Figure 75). By looking at the right-hand column of the Coefficients table, it is clear that independent variable NumberOfMissingClasses is shown to have a significant relationship (i.e, .000) with the dependent variable, TotalPointsEarned. This result could be interpreted as meaning that the more classes a student misses, the lower their total class points earned will be. Obviously this is a contrived dataset, however the two authors are also professors and we would like to believe that this is true!

4. Now that the regression is complete, it is time to store the results. Save the SPSS Statistical Viewer file as 06_MissedClassesAndPointsEarned.spo; save the data file as 06_MissedClassesAndPointsEarned.sav. Close SPSS.

Figure 75: Regression Results in SPSS Statistics Viewer

\begin{tabular}{|c|c|c|c|c|c|c|c|c|}
\hline \multicolumn{6}{|c|}{ Model Summary } & & & \\
\hline Model & $\mathrm{R}$ & R Square & \multicolumn{2}{|c|}{$\begin{array}{c}\text { Adjusted R } \\
\text { Square }\end{array}$} & $\begin{array}{l}\text { Std. Error of } \\
\text { the Estimate }\end{array}$ & & & \\
\hline 1 & $.950^{\mathrm{a}}$ & .902 & & .894 & 45.226 & & & \\
\hline \multicolumn{9}{|c|}{ a. Predictors: (Constant), NumberofmissedClasses } \\
\hline \multicolumn{9}{|c|}{ ANOVA ${ }^{b}$} \\
\hline \multicolumn{2}{|l|}{ Madel } & \multicolumn{2}{|c|}{$\begin{array}{c}\text { Sum of } \\
\text { Squares }\end{array}$} & df & Mean Square & $\mathrm{F}$ & Sig. & \\
\hline \multirow[t]{3}{*}{1} & Regression & \multirow{2}{*}{\multicolumn{2}{|c|}{$\begin{array}{r}227176.361 \\
24545.068\end{array}$}} & 1 & 227176.361 & 111.066 & $.000^{a}$ & \\
\hline & Residual & & & 12 & 2045.422 & & & \\
\hline & Total & 25172 & & 13 & & & & \\
\hline \multicolumn{9}{|c|}{ a. Predictors: (Constant), NumberOfMissedClasses } \\
\hline \multicolumn{9}{|c|}{ b. Dependent Variable: TotalPointsEarned } \\
\hline \multicolumn{9}{|c|}{ Coefficients $^{a}$} \\
\hline \multirow[b]{2}{*}{ Model } & & & \multicolumn{3}{|c|}{ Unstandardized Coefficients } & $\begin{array}{l}\text { Standardized } \\
\text { Coefficients }\end{array}$ & \multirow[b]{2}{*}{$\mathrm{t}$} & \multirow[b]{2}{*}{ Sig. } \\
\hline & & & & $\mathrm{B}$ & Std. Error & Beta & & \\
\hline \multirow[t]{2}{*}{1} & (Constant) & & & 854.836 & 18.765 & & 45.556 & .000 \\
\hline & $\begin{array}{l}\text { Numberofm } \\
\text { Classes }\end{array}$ & iissed & & -78.433 & 7.442 & -.9 & -10.539 & .000 \\
\hline
\end{tabular}




\section{CONCLUSION}

The purpose of this paper was to help researchers deal with ill-behaved data through the use of relational database software, with particular focus on QBE (Query-By-Example). We believe we have accomplished this goal through a demonstration consisting of the following steps:

- $\quad$ How to import a misconfigured dataset from MS Excel into an MS Access table.

- $\quad$ Five examples of how to use MS Access' QBE to reconfigure datasets.

- How to import a reconfigured dataset from MS Access into SPSS.

The broader purpose of this paper is to demystify database software and encourage the reader to learn more about the usefulness of database tools and techniques in their own field of expertise. To this end, we have included in our reference list a number of database theory and MS Access texts that could be used for further explanations of database topics. We hope that they will prove helpful.

\section{AUTHOR INFORMATION}

Dr. Paul J. Lazarony is an Associate Professor of Information Systems at California State University, Northridge. His current research interests are assessment, database, enterprise-wide systems, and IT project management. He received his $\mathrm{PhD}$ in Computer Technology and Business Education from the Ohio State University.

Dr. Donna A. Driscoll is a Professor of Information Systems at California State University, Northridge. Her current research interests are database, information assurance, and web-based systems development. She received her $\mathrm{PhD}$ in Business Administration (Accounting and Research Statistics) from the University of Southern California.

\section{REFERENCES}

1. $\quad$ Groh, M. et al. (2007). Access 2007 Bible, Wiley Publishing, Indianapolis, IN.

2. Hoffer, A. and Prescott, M. (2009). Modern Database Management, Ninth Edition, Pearson Prentice Hall, Upper Saddle River, NJ.

3. Kroenke, D. and Auer, D. (2010). Database Concepts, Fourth Edition, Prentice Hall, Boston, MA.

4. Kroenke, D. and Auer, D. (2010). Database Processing: Fundamentals, Design, and Implementation, Eleventh Edition, Prentice Hall, Boston, MA.

5. $\quad$ Post, G. (2005). Database Management Systems, Third Edition, McGraw-Hill, Boston, MA.

6. Rob, P. and Coronel, C. (2007). Database Systems: Design, Implementation and Management, Seventh Edition, Thomson Course Technology, Boston, MA.

7. Shelly, G. and Cashman, T. (2007). Microsoft Office Access 2007: Comprehensive Concepts and Techniques, Thomson Course Technology, Boston, MA. 